\title{
PUBLICATIONS
}

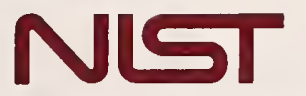

National Institute of Standards and Technology

Technology Administration, U.S. Department of Commerce

NIST Technical Note 1534

\section{Uncertainty Analysis of Radar Cross Section Calibrations at Etcheron Valley Range}

Lorant A. Muth

Dale M. Diamond

John A. Lelis 



\section{Uncertainty Analysis of Radar Cross Section Calibrations at Etcheron Valley Range}

Lorant A. Muth

Dale M. Diamond

John A. Lelis

Electromagnetics Division

Electronics and Electrical Engineering Laboratory

National Institute of Standards and Technology

Boulder, CO 80305

September 2004

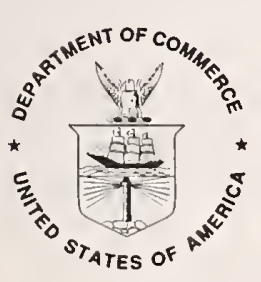

U.S. Department of Commerce

Donald L. Evans, Secretary

Technology Administration

Phillip J. Bond, Under Secretary for Technology

National Institute of Standards and Technology

Arden L. Bement, Jr., Director 
Certain commercial entities, equipment, or materials may be identified in this document in order to describe an experimental procedure or concept adequately. Such identification is not intended to imply recommendation or endorsement by the National Institute of Standards and Technology, nor is it intended to imply that the entities, materials, or equipment are necessarily the best available for the purpose.

National Institute of Standards and Technology Technical Note 1534 Natl. Inst. Stand. Technol. Tech. Note 1534, XX pages (September 2004) CODEN: NTNOEF 


\title{
UNCERTAINTY ANALYSIS \\ OF RADAR CROSS SECTION CALIBRATIONS AT ETCHERON VALLEY RANGE* ${ }^{\dagger}$
}

\author{
Lorant A. Muth ${ }^{1}$, \\ Dale M. Diamond ${ }^{2}$, and John A. Lelis ${ }^{3}$
}

\begin{abstract}
The Etcheron Valley Range, Naval Air Warfare Center, Weapons Division at China Lake, CA conducts monostatic and bistatic measurements of radar cross section on various targets of interests to DoD. This document, which follows general guidelines proposed by the National Institute of Standards and Technology, discusses the assessment of uncertainty in calibrations of radar cross section at the Etcheron Valley Range, NAWC-WD, a division of NAVAIR located at China Lake, CA.
\end{abstract}

Key words: calibration uncertainties; radar cross section measurements; RCS; uncertainty analysis

\section{INTRODUCTION}

The National Institute of Standards and Technology (NIST) has established a framework for estimating uncertainties in radar cross section (RCS) measurements [1]. In this study we follow the basic outline and format presented in [1] to determine the calibration uncertainty at the RCS facilities of the Etcheron Valley Range, NAVAIR, Naval Air Warfare Center, Weapons Division (NAWC-WD), China Lake, CA.

The Etcheron Valley Range (EVR) measures radar cross section of various targets provided by United States military and civilian customers. The EVR currently operates coherent RCS measurement systems capable of measuring in the VHF, UHF, L, S, C, X, Ku and Ka bands (see Appendix A, Table A.1). The North 40 look-down slant range and the South 40 outdoor bounce range (see Figures $1.1-1.4$ ) are two fully operational sytems. Typical targets measured on these ranges are unmanned aerial vehicles, airplane components, aircraft models and components, ship models and components, missiles and tactical ballistic missiles, very low-observable (vlo) articles, reentry vehicles, ground vehicles, rockets and rocket plumes. The North 40 range can also be used to simulate the ocean environment for measuring the RCS of ship models and components.

The South 40 range can be configured to measure the low-frequency bistatic RCS of targets

*Distribution authorized to DoD components and U.S. Dept. of Commerce only; Administrative or Operational Use; June 2004.

${ }^{\dagger}$ A U.S. Government publication, not subject to copyright in the United States.

${ }^{1}$ NIST, Electromagnetics Division, Boulder, CO

${ }^{2}$ Consultant, Etcheron Valley Range, China Lake, CA

${ }^{3}$ Etcheron Valley Range, China Lake, CA 


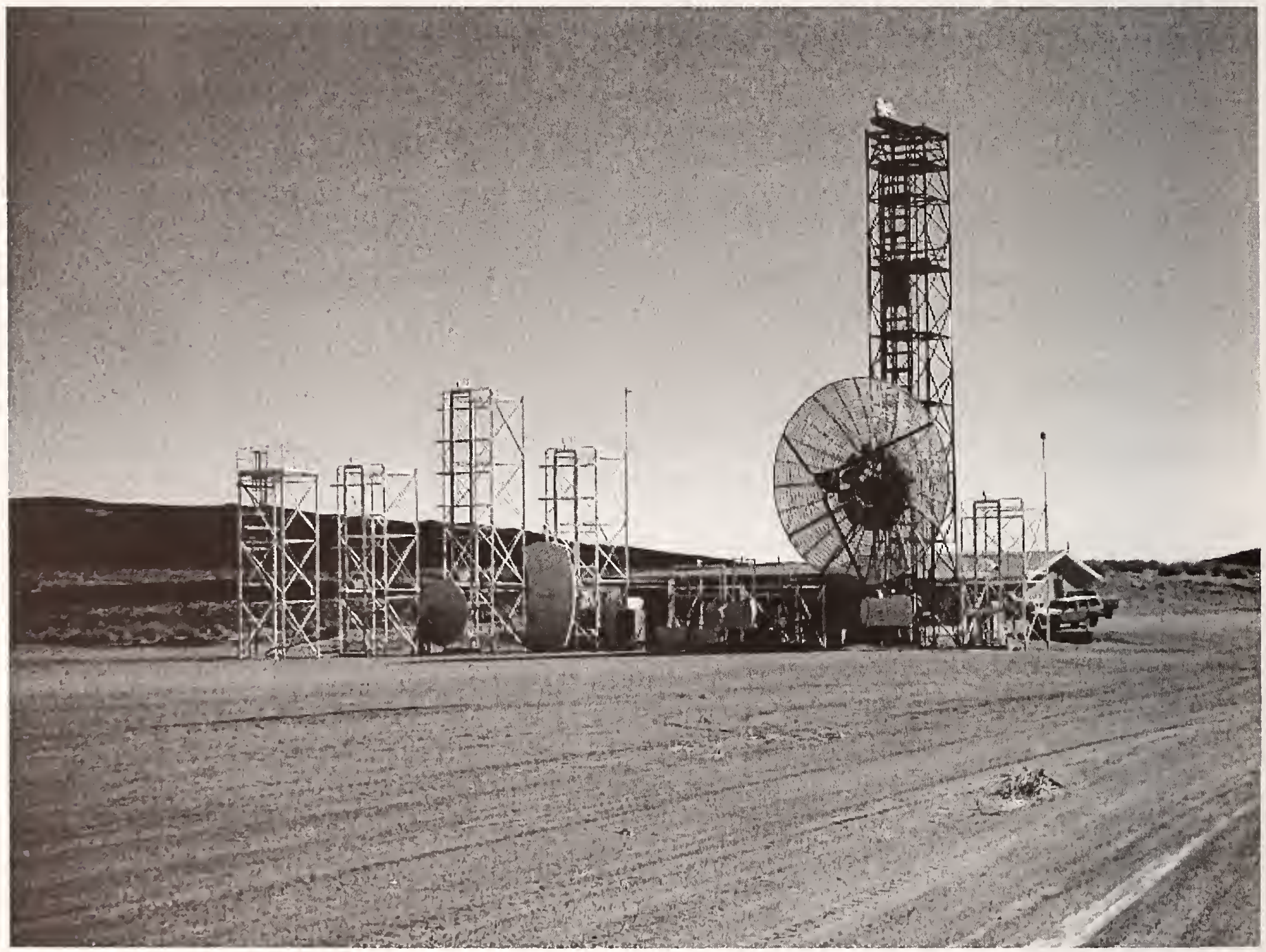

Figure 1.1. The South 40 outdoor bounce-range antenna farm capable of making coherent monostatic RCS measurements in the VHF, UHF, L, S, C, X, Ku, and Ka bands. Additionally, it can be configured to make coherent bistatic RCS measurements at the VHF, UHF, and L-band frequencies. 


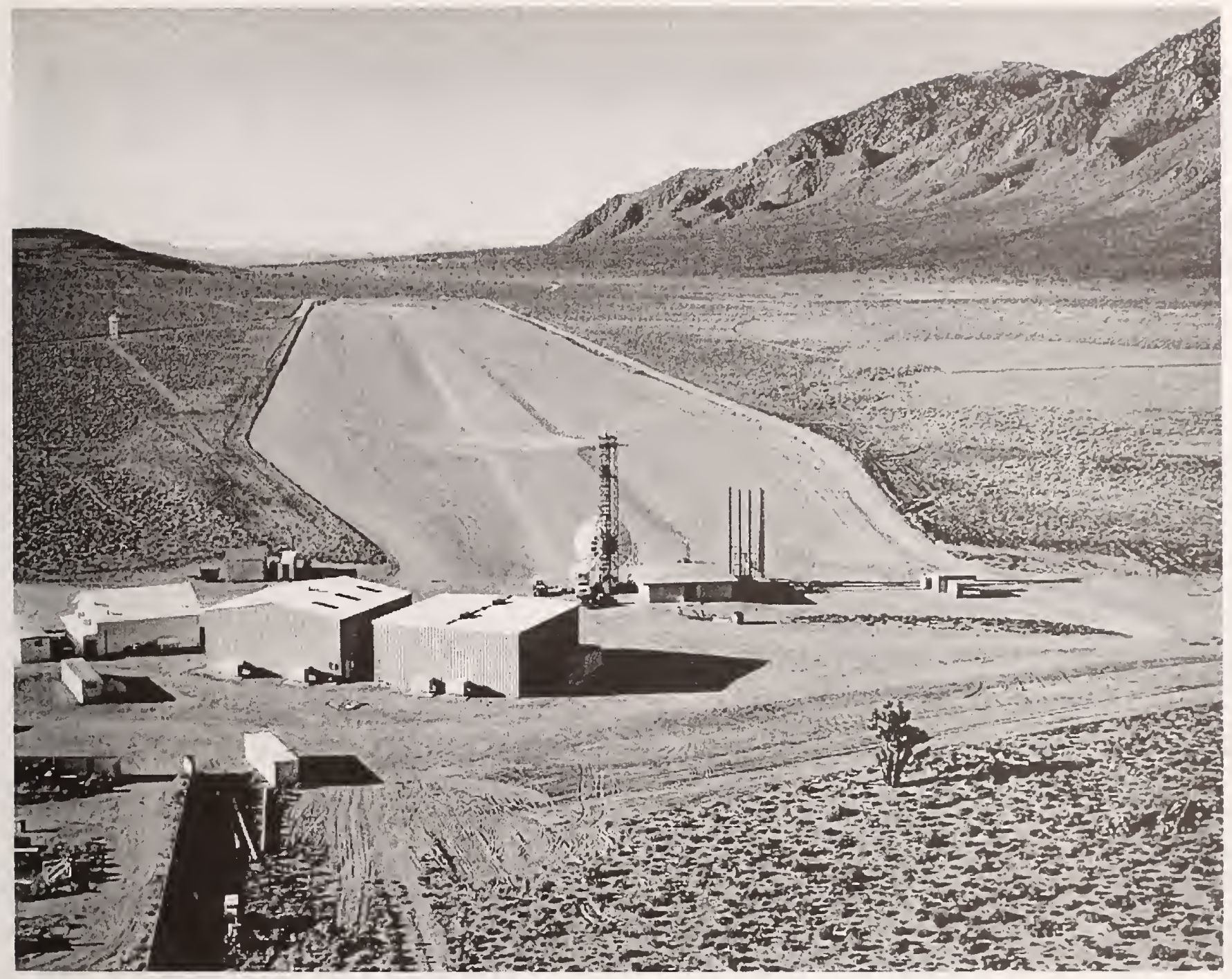

Figure 1.2. Rear view of the South 40 antenna farm and the measurement range. Monostatic measurements on full-scale targets can be made at various ranges, for example, at $213 \mathrm{~m} \mathrm{(700} \mathrm{ft),}$ $610 \mathrm{~m}(2000 \mathrm{ft}), 1220 \mathrm{~m}(4000 \mathrm{ft})$ and $1524 \mathrm{~m} \mathrm{(4000} \mathrm{ft).} \mathrm{A} \mathrm{target} \mathrm{at} 274 \mathrm{~m}(900 \mathrm{ft})$ is used for bistatic RCS measurements at VHF, UHF, and L-band frequencies. 


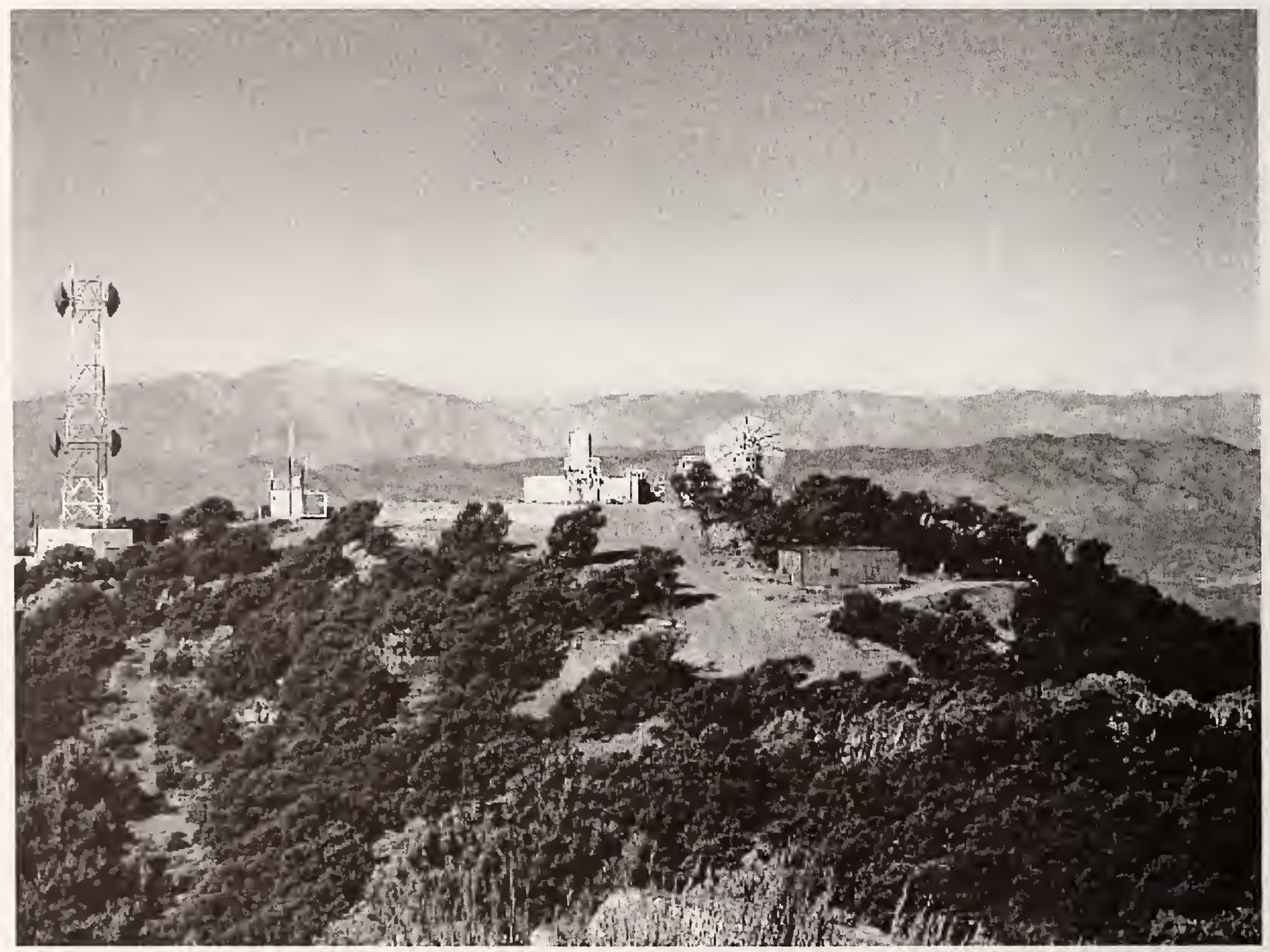

Figure 1.3. The Parrot peak look-down range measurement facility used to measure targets deployed on the North 40 tilt deck or wet site in Figure 1.4. The look-down angle is 10 degrees and the slant range is $5182 \mathrm{~m}(17,000 \mathrm{ft})$. Measurements have been made in the $\mathrm{S}, \mathrm{X}, \mathrm{Ku}, \mathrm{Ka}$, and $\mathrm{W}$ bands. 


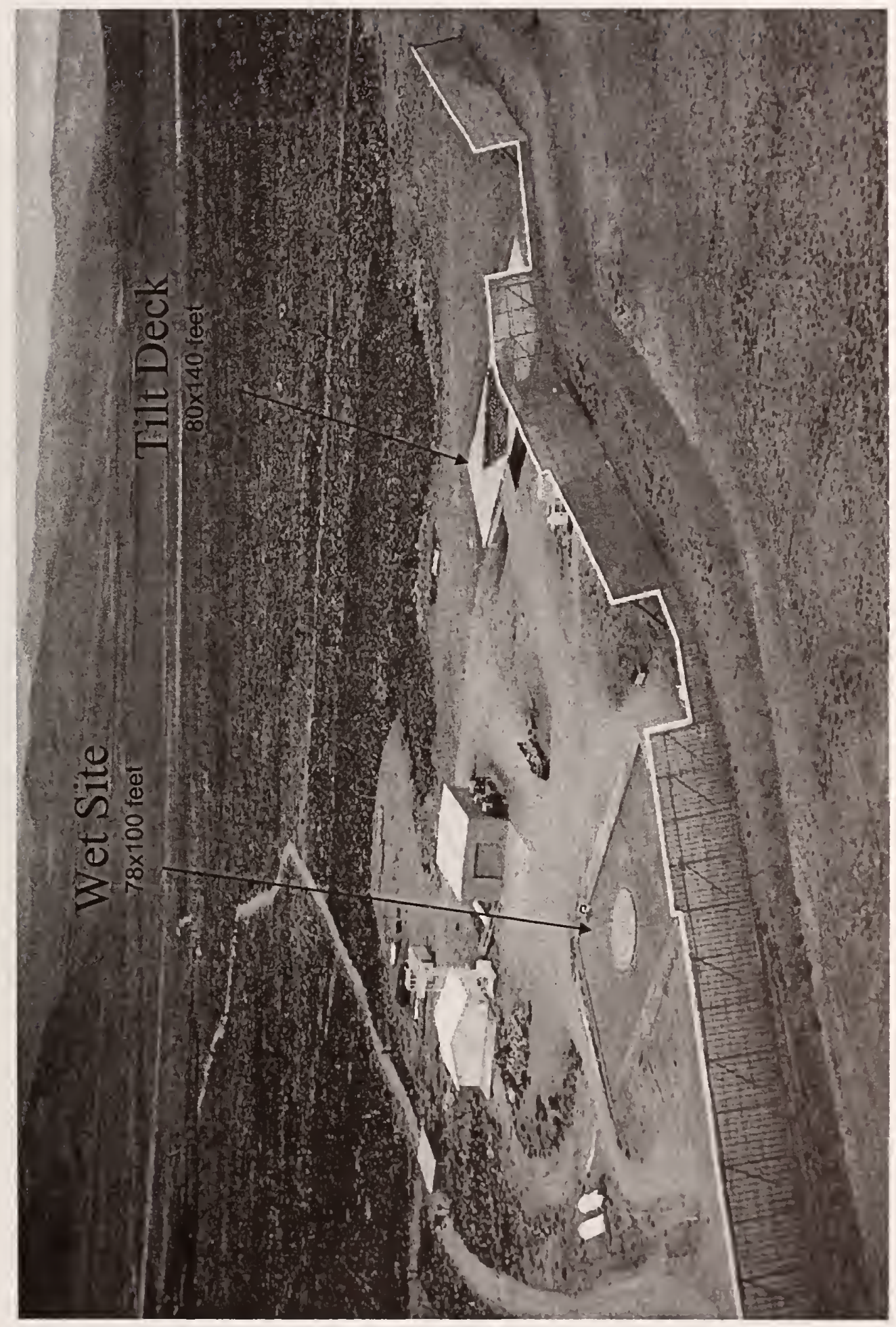

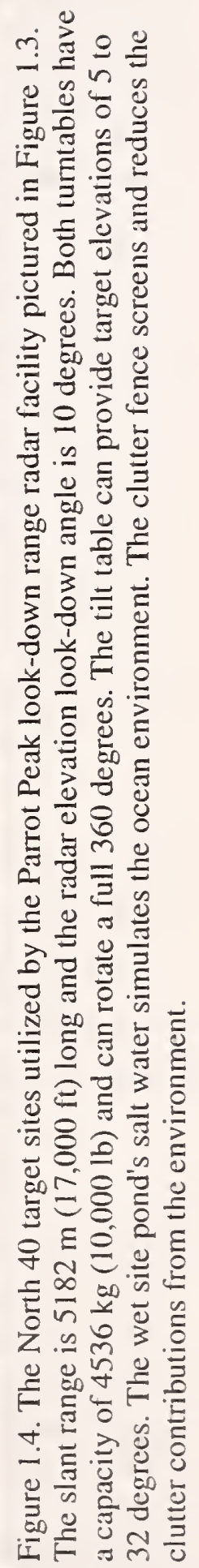


at VHF, UHF and L-band frequencies. This work evolved from earlier coherent bistatic measurement systems that operated from 30 to $150 \mathrm{MHz}$ and at $\mathrm{X}$ band. The capability to perform bistatic measurements using the system developed at EVR has been demonstrated recently by successfully completing measurements at various bistatic angles, for example, $35^{\circ}, 70^{\circ}, 120^{\circ}$ and $145^{\circ}$. The uncertainties of the bistatic measurements will be the subject of a future study.

In this study, we intend to provide procedures for estimating all sources of uncertainties of the South 40 RCS measurement system. We consider the uncertainties in the monostatic calibration of the system using standard RCS calibration artifacts as well as the uncertainties in the monostatic measurement of the RCS of test targets. Calibration and measurement uncertainty depends on the operational parameters of a RCS range and is specific to the parameters in effect at the time of measurement (see Appendix A, Tables A.2 and A.3). Typical operating conditions and parameters for RCS measurements are assumed for the analysis presented in this report. In our measurement-based approach, a worst-case scenario, wherein we tend to overestimate the uncertainty, is assumed. Where appropriate, we explicitly state any assumptions made to support our uncertainty analysis.

\section{REPORTING MEASUREMENT UNCERTAINTY}

Here we follow closely the discussion of uncertainty given in [1]. A measurement of $\sigma$ (RCS) should be quantified by a statement of uncertainty

$$
\sigma=\sigma_{0} \pm \Delta \sigma
$$

where $\sigma_{0}$ indicates the best RCS estimate, and $\Delta \sigma>0$ is a reasonable bound for the measurement error. Error bounds need not be symmetric:

$$
\sigma_{0}+\Delta \sigma_{+}>\sigma>\sigma_{0}-\Delta \sigma_{-} .
$$

For simplicity, we will continue to use eq (1) with $\Delta \sigma=\max \left(\Delta \sigma_{+}, \Delta \sigma_{-}\right)$.

Uncertainties are also stated logarithmically

$$
\Delta \sigma_{ \pm}(\mathrm{dB}) \equiv \pm 10 \log \left(1 \pm \frac{\Delta \sigma}{\sigma_{0}}\right) .
$$

As before, we will often use symmetric bounds. In this case, since $\Delta \sigma_{-}(\mathrm{dB}) \geq \Delta \sigma_{+}(\mathrm{dB})$,

$$
\Delta \sigma(\mathrm{dB}) \equiv \Delta \sigma_{-}(\mathrm{dB})=-10 \log \left(1-\frac{\Delta \sigma}{\sigma_{0}}\right)
$$

or equivalently,

$$
\Delta \sigma(\mathrm{dB}) \equiv \Delta \sigma_{-}(\mathrm{dB})=-20 \log \left(1-\frac{\Delta S}{S_{0}}\right),
$$

where $S$ is the measured electric field signal under consideration. Asymmetric bounds may be given when reporting larger logarithmic uncertainties. For example, if $\Delta \sigma / \sigma_{0}=1$, then $\Delta \sigma_{+}(\mathrm{dB}) \approx 3$, while $\Delta \sigma_{-}(\mathrm{dB})=-\infty$. 
The individual sources of uncertainty (called components of uncertainty) should be selected so that they are at least approximately independent. Sample NAVAIR-NAWC-WD uncertainty tables are shown in Table 2.1, where the uncertainty estimates are reported logarithmically. The top table shows uncertainties related to measurement of a test target; the bottom table shows uncertainties associated with the primary calibration target. These component uncertainties are identified in the tables with numbers that reference the sections of this report ${ }^{1}$ where the corresponding uncertainty is discussed. The last entry of each table shows the overall uncertainty, which is the root sum of squares (RSS) of the component uncertainties.

In general, an entry of "neg." in the tables would indicate that, in our opinion, the effect is unimportant (which could be defined operationally as a magnitude less than about 0.1 dB). An entry of "n.a." (not applicable) would indicate that this source is not considered a factor in the current evaluation (integration error due to target motion, for example, is not applicable when the target is stationary).

The entries are an attempt to represent typical NAVAIR-NAWC-WD uncertainties for the RCS system configuration and parameters given in Appendix A. The reader should understand, however, that actual uncertainties depend strongly on the particular measurement configuration. Again, an uncertainty analysis should be performed for each measurement situation. For example, we need separate tables as a function of signal level for each frequency band.

The method of uncertainty combination is RSS. That is, the overall uncertainty $\Delta \sigma$ is calculated as

$$
\left(\frac{\Delta \sigma}{\sigma_{0}}\right)^{2}=\sum_{i}\left(\frac{\Delta \sigma_{i}}{\sigma_{0}}\right)^{2}
$$

where $\Delta \sigma_{i}$ are the (independent) components of uncertainty. Note that relative (not logarithmic) uncertainty is used in the calculation of eq (5). That is, from eq (4a),

$$
\frac{\Delta \sigma}{\sigma_{0}}=1-10^{-\Delta \sigma_{d B} / 10}
$$

or from eq $(4 \mathrm{~b})$,

$$
\frac{\Delta S}{S_{0}}=1-10^{-\Delta \sigma_{d B} / 20}
$$

In general, the combination of individual uncertainties using RSS, as in eq (5), can be thought of as the resultant of independent uncertainty vectors in a multidimensional space. This also agrees with the well known formula that the uncertainties in products and quotients of measured quantities propagate, in general, as the RSS of fractional errors as shown in eq (5) [2]. The usual method of derivation is to assume that the individual uncertainties $\Delta \sigma_{i}$ are small. However, we can show that this method of combination of uncertainties is also valid if the fractional errors $\Delta \sigma_{i} / \sigma_{i}<<1$. In practice, the measured fractional errors in RCS calibration and measurements satisfy this condition. Possible exceptions might

${ }^{1}$ Sections here generally follow the sections of NISTIR 5019 [1] 
occur in extremely low-observable measurements, where the the target RCS is comparable to the system noise RCS. However, such measurements are not considered in this report. The component uncertainties in any uncertainty table can be either measurement-based, that is, well defined measurement procedures are used to determine their magnitudes, or the RSS of a number of lower level components that are summarized in separate uncertainty tables. An example is the second table in Table 2.1, which gives uncertainties associated with the standard target calibration. The overall calibration uncertainty (line 4.14) is included as a contribution to the test target measurement uncertainty (line 3.13, Reference RCS).

Measurement-based uncertainties usually reflect a worst-case estimate. The term worst case should be operationally defined. Procedures that estimate worst-case uncertainty will generally reflect practical experience. Statistically, we assign such worst-case estimates to represent two standard deviations, although the actual error distribution in question is unknown. Loosely speaking, a metrologist is $95 \%$ confident [2] that the actual error falls within the worst-case uncertainty bounds.

\section{TEST TARGET UNCERTAINTIES}

The radar equation [3-5] can be expressed as a ratio of test target RCS to calibration target RCS:

$$
\frac{\sigma_{0}}{\sigma_{s}}=\left(\frac{R}{R_{s}}\right)^{4}\left(\frac{G_{s}}{G}\right)^{2}\left(\frac{f}{f_{s}}\right)^{2} \frac{P_{t s}}{P_{r s}} \frac{P_{r}}{P_{t}}
$$

where

$$
\begin{aligned}
& \sigma=\text { radar cross section }\left[\mathrm{m}^{2}\right] \\
& R=\text { range (distance) }[\mathrm{m}], \\
& G=\text { antenna gain, } \\
& f=\text { frequency }\left[\mathrm{s}^{-1}\right] \\
& P_{t}=\text { transmitted (delivered) power }[\mathrm{W}], \\
& P_{r}=\text { received power }[\mathrm{W}] .
\end{aligned}
$$

The subscript $s$ identifies quantities associated with the standard calibration target. For example, we allow the possibility that $G_{s} / G \neq 1$, as could result from pointing errors (which could be significant on dynamic, but negligible on static, ranges).

Equivalently, the expression for the measured RCS in eq (7a) can also be written as [3-5]

$$
\sigma_{0}=\left|\frac{E}{E_{s}}\right|^{2} \sigma_{s}
$$

where $E$ is the measured electric field. In practice, especially at indoor ranges, background subtraction techniques can be used to improve the uncertainty in $E$. However, a careful study of this technique has yet to be completed.

In this report we do not actually determine a specific target's measurement uncertainties. In Table 2.1a we assumed arbitrary values for test-target uncertainties to illustrate the calculation of an overall RCS uncertainty. However, we plan to publish soon a separate document detailing the determination of target uncertainties for a specific target. 
Table 2.1. Etcheron Valley Range Estimated RCS Uncertainties at $11.3 \mathrm{GHz}$.

$\begin{array}{llr}\text { A. } & \text { Test target uncertainties } & {[\mathrm{dB}]} \\ 3.1 & \text { Average illumination } & 0.40 \\ 3.2 & \text { Background-target interaction } & 0.50 \\ 3.3 & \text { Cross polarization } & 1.00 \\ 3.4 & \text { Drift } & 0.30 \\ 3.5 & \text { Frequency } & 0.16 \\ 3.6 & \text { Integration } & 0.10 \\ 3.7 & \text { I-Q imbalance } & 0.10 \\ 3.8 & \text { Near field } & 0.20 \\ 3.9 & \text { Noise-background } & 0.35 \\ 3.10 & \text { Nonlinearity } & 0.25 \\ 3.11 & \text { Range } & 0.01 \\ 3.12 & \text { Target orientation } & \text { na } \\ 3.13 & \text { Reference RCS (4.14) } & 0.71 \\ 3.14 & \text { Combined uncertainty (RSS) } & 1.20 \\ & & -1.70\end{array}$

B. Calibration target uncertainties [dB]

4.1 Average illumination

4.2 Background-target interaction $\quad 0.25$

4.3 Cross polarization 0.3

$\begin{array}{lll}4.4 & \text { Drift } & 0.15\end{array}$

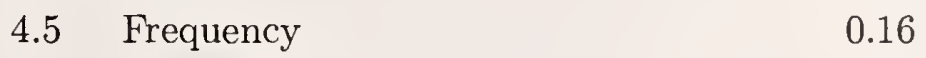

4.6 Integration 0

4.7 I-Q imbalance 0.1

4.8 Near field 0

4.9 Noise-background 0.35

4.10 Nonlinearity 0.25

4.11 Range 0

4.12 Target orientation $\quad 0.10$

4.13 Reference RCS (computation) $\quad 0.10$

4.14 Combined uncertainty (RSS) 0.71 


\section{CALIBRATION TARGET UNCERTAINTIES}

In this section we determine the calibration uncertainty at the South 40 Etcheron Valley Range (EVR). The various uncertainty contributions listed in Table 2.1 are individually discussed in the following subsections.

We briefly characterize the components of calibration uncertainty and the measurement procedures used to determine them. Some details on the system parameters are provided in the appendices. All components of the calibration uncertainty are listed in Table 2.1(b). At EVR the standard cylinder $22.86 \mathrm{~cm}$ by $10.67 \mathrm{~cm}$ (9.0 in by $4.2 \mathrm{in}$ ) was used to calibrate the RCS system for uncertainty measurements using the four standard cylinders (see Figures 4.1 and 4.2). Manufacturing imperfections in the standard cylinder artifacts (see Table 4.1) and errors in their computed RCS values contribute to the overall calibration uncertainty. As shown in eq (7b) the computed value together with the measured RCS of the calibration artifact are used to determine the calibration constant with stated uncertainties. The calibration uncertainty is then propagated into the measurement uncertainties (see Table 2.1) to obtain the overall uncertainty of a RCS measurement.

We utilized measurements made on all the four cylinders to determine the calibration uncertainty valid at $11.3 \mathrm{GHz}$. This uncertainty analysis is valid in the RCS range of -5 to $-17 \mathrm{dBsm}$, as indicated in Table 4.1. A sphere of $30.48 \mathrm{~cm}$ (12 in) diameter can be used to provide additional data and a quick check on the calibration using the cylinders. Such a sphere mounted on a styrofoam column is shown in Figure 4.3. The cylinders were mounted similarly. The styrofoam column was located at $R_{s}=610 \mathrm{~m}$ from the antenna. Measurements were made at 2 to $18 \mathrm{GHz}$; we will concentrate on measurements at $11.3 \mathrm{GHz}$ (for the system parameters, see Appendix B, Tables B.1, B.2 and B.3). Our uncertainty analysis is valid for the specific parameters and system configuration used in these measurements. In general, changes in system parameters and configurations will change the system uncertainty.

\begin{tabular}{|c|c|c|c|c|c|}
\hline \multicolumn{6}{|c|}{ Table 4.1. The EVR Calibration Cylinders. ${ }^{1}$} \\
\hline Designation $^{2}$ & Radius $^{3}$ & Tolerance & Height & Tolerance & Computed $\mathrm{RCS}^{4}$ \\
\hline 375 & 9.525 & \pm 0.0005 & 4.445 & \pm 0.0005 & -16.5 \\
\hline 450 & 11.43 & \pm 0.0005 & 5.145 & \pm 0.0005 & -14.2 \\
\hline 750 & 19.05 & \pm 0.0005 & 8.890 & \pm 0.0005 & -7.45 \\
\hline 900 & 22.86 & \pm 0.0005 & 10.668 & \pm 0.0005 & -5.12 \\
\hline \multicolumn{6}{|c|}{$\begin{array}{l}{ }^{1} \text { Cylinders were made at NIST using Al } 6061 \text { to a surface } \\
\text { finish of } 32 \text { microinches AA or better. }\end{array}$} \\
\hline \multicolumn{6}{|c|}{$\begin{array}{l}2 \text { Indicates the nominal radius in inches times } 100 \\
{ }^{3} \text { Dimensions and tolerances (in } \mathrm{cm} \text { ) were determined } \\
\text { using a Mitutoyo CMM at the NIST dimensional analysis } \\
\text { laboratory. An analysis report is available at the EVR } \\
\text { RCS measurement range. } \\
{ }^{4} \text { Computed for the HH polarization (dBsm) to determine } \\
\text { calibration constants. For VV polarization, see Figure } 4.2\end{array}$} \\
\hline
\end{tabular}




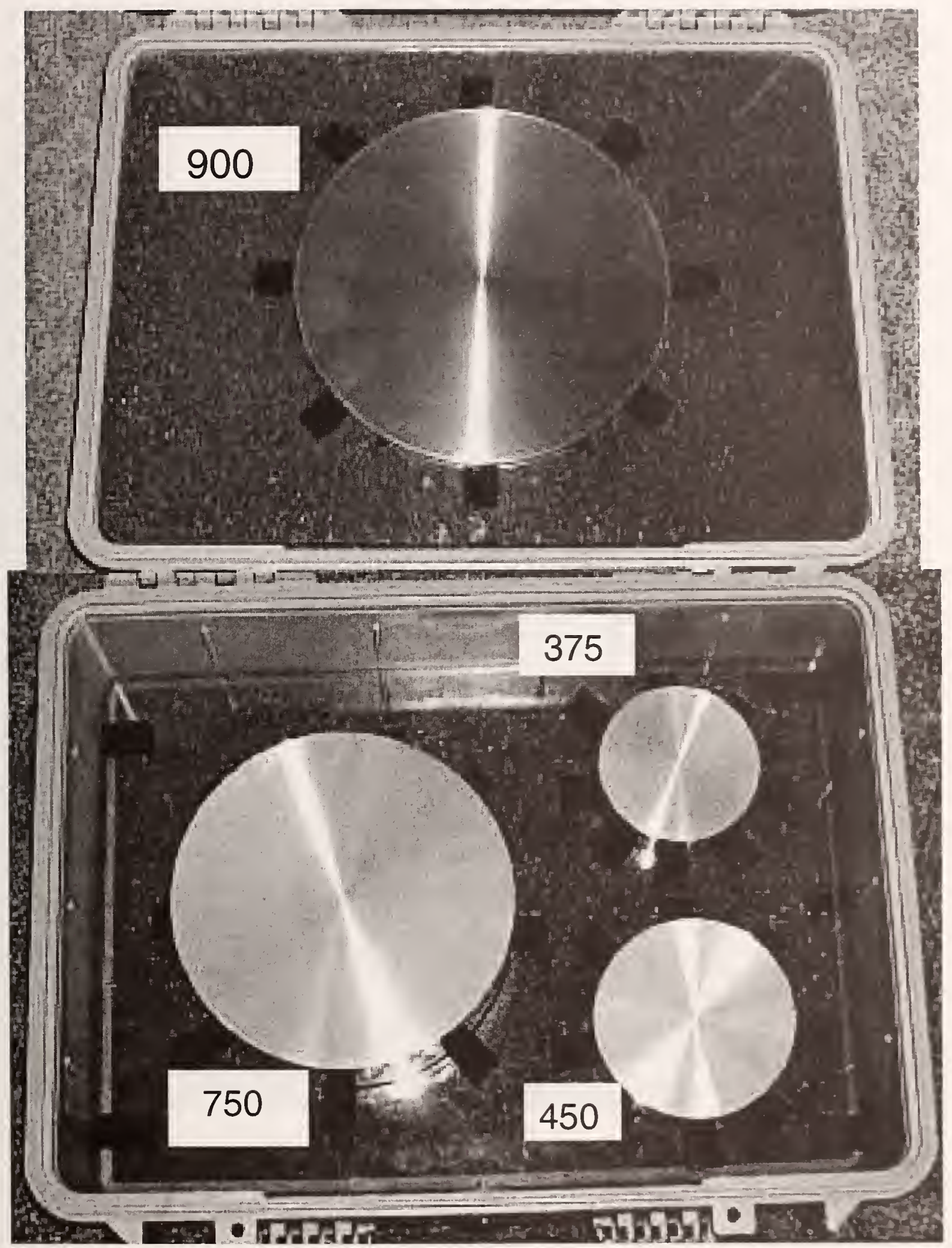

Figure 4.1. The standard cylinder set adopted by the RCS community used to calibrate the EVR RCS measurement system from 2 to $18 \mathrm{GHz}$. The cylinders are designated according to their diameters (in inches $x$ 100). For dimensions with tolerances and computed cross sections, see Table 4.1 . 


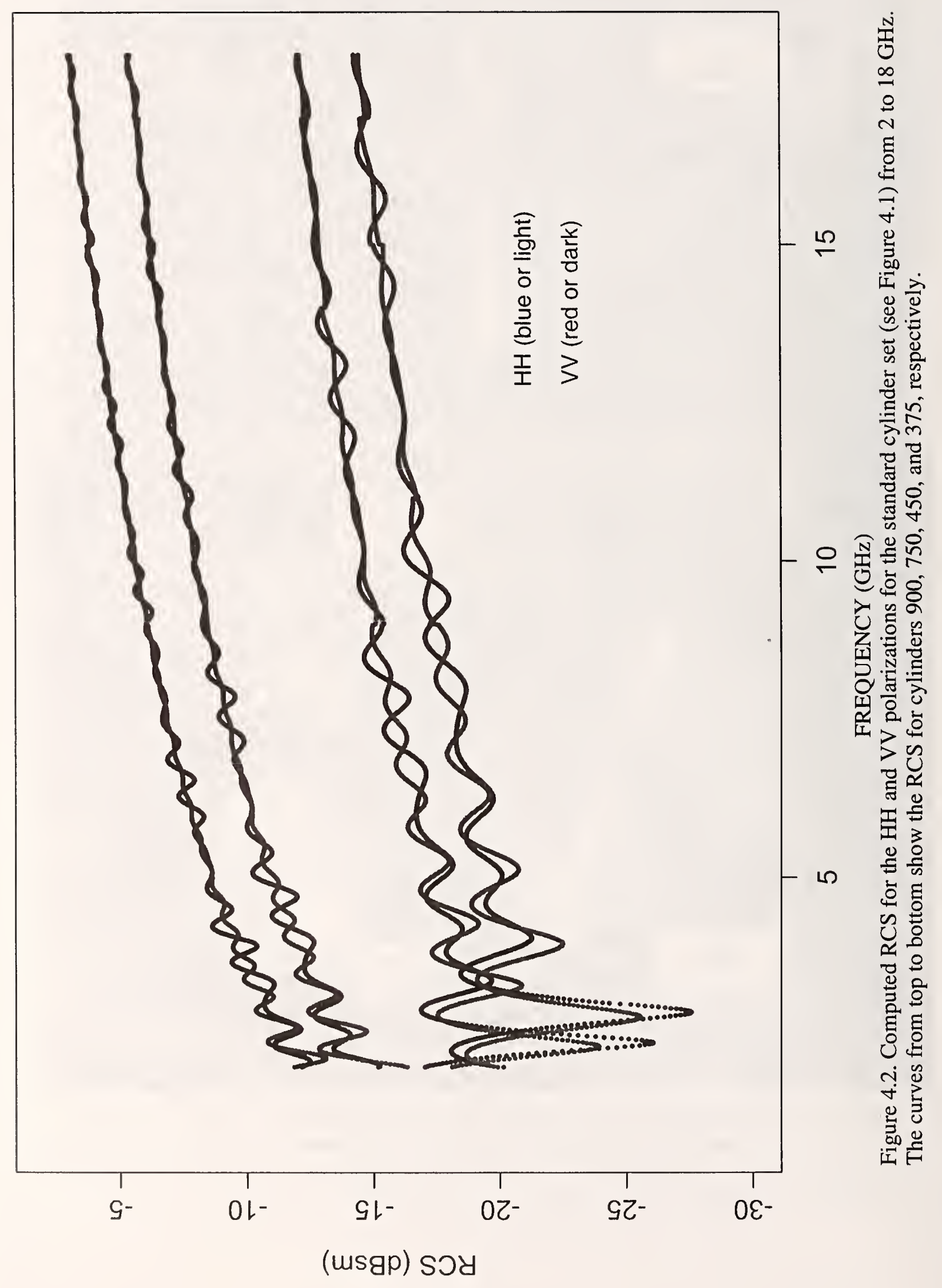




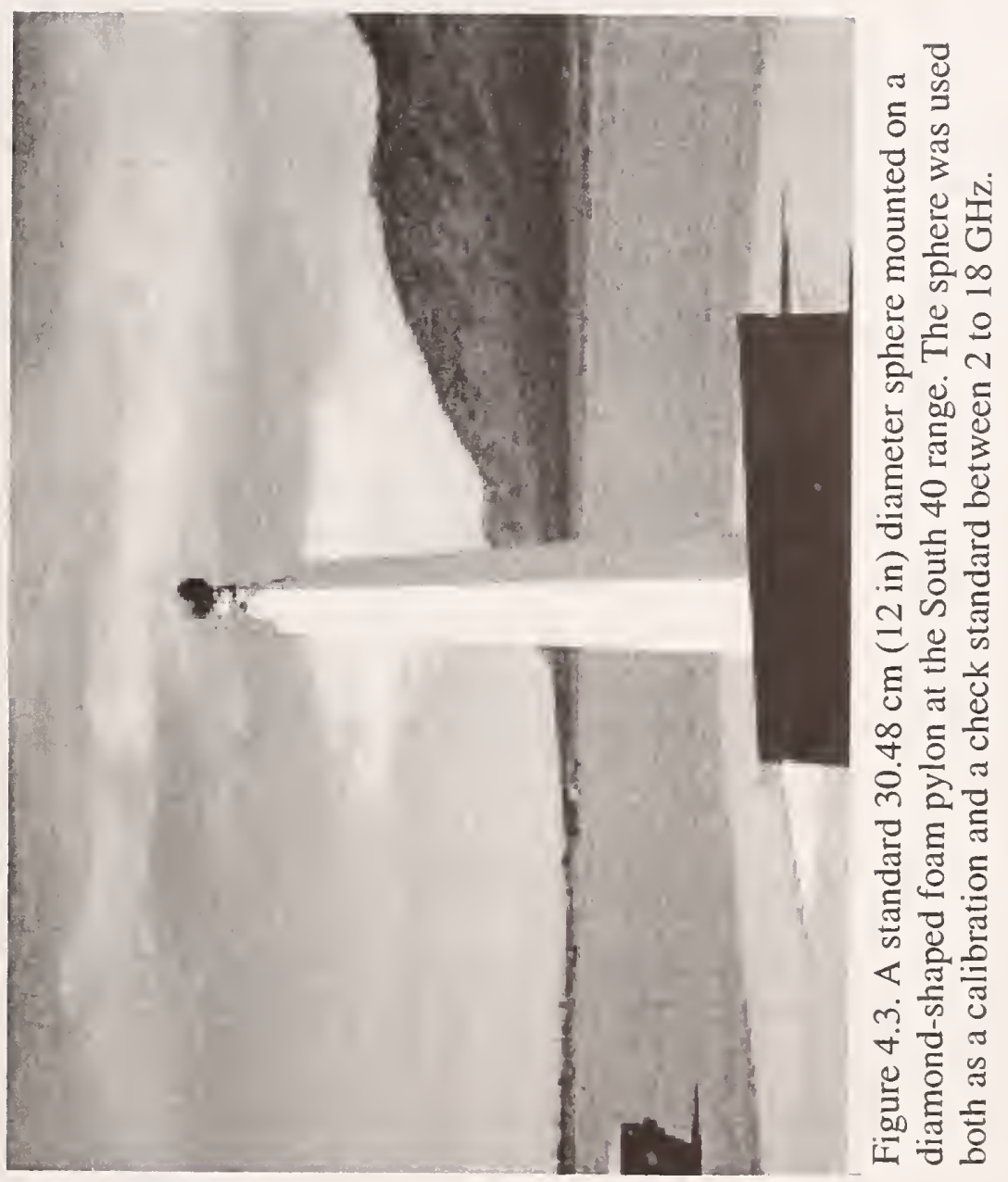




\subsection{AVERAGE ILLUMINATION}

In Figure 4.1.1 we show the uncalibrated RCS of a small corner reflector as a function of height in the neighborhood of the target measurement height. Since the response of a corner reflector is insensitive to small changes in orientation, we assume that the observed variations in RCS in large part demonstrate the nonplanar illumination of the target volume. This is the result of interference pattern in the vertical direction produced by the direct illumination and indirect ground-bounce rays. However, smaller variations in gain (a function of direction) are also included. A large variation of approximately $6 \mathrm{~dB}$ in RCS is observed over the scan heights ranging from approximately 200 to $600 \mathrm{~cm}$. We assume that the variation in the illumination pattern in the horizontal direction is due primarily to the variation in the antenna pattern, that is, the horizontal direction does not exhibit significant interference effects. Hence, we estimate illumination uncertainty using field variations in the vertical direction.

The radius and height of the largest cylindrical calibration artifact used from 2 to 18 $\mathrm{GHz}$ are approximately 23 and $11 \mathrm{~cm}$, respectively (see Table 4.1, Figures 4.1 and 4.2). This cylinder is located at the point of maximum illumination during calibration, which is approximately at $416 \mathrm{~cm}$. A maximum variation in illumination of $<0.2 \mathrm{~dB}$ in RCS is observed in the range of 416 to $440 \mathrm{~cm}$.

Obviously, illumination uncertainty depends on both the target and the target height. We assumed that the upper bound uncertainty due to nonplanar illumination for an extended target can be determined as for a small target. Then, from Figure 4.1.1, a target $200 \mathrm{~cm}$ high would be subject to an illumination uncertainty of approximately $1 \mathrm{~dB}$.

To determine the illumination uncertainty for a target extended in the horizontal direction, we need horizontal field plots. Currently, such plots are not available.

Estimated uncertainty: $\Delta \sigma(\mathrm{dB})=0.2$

\subsection{BACKGROUND-TARGET INTERACTIONS}

This contribution to RCS uncertainty comes from the interaction between the calibration artifact (cylinder or sphere) or target and the supporting structure. The support is usually a polyfoam column or pylon.

Background-target interaction is strongest where the target and mounting mechanism are closely coupled, due to physical proximity. Under such conditions, time gating is of no value, and the interaction needs to be included as an uncertainty in the measurement. In general, computer simulations could provide a good estimate of the magnitude of interaction. However, in a measurement-based approach, we can determine an upper-bound estimate by making a few general assumptions. Such estimates can be verified using computer simulations for complex targets; for example, full-scale airplanes.

To estimate the interaction uncertainty we observe that the target-mount interaction is a complex sum of signals that have followed all possible signal paths between the transmitting radar, the target, the pylon, and the receiving radar. For example, $A_{R} P T A_{T}$ denotes the signal path from the transmitting radar to the target, then to the pylon and back to the 


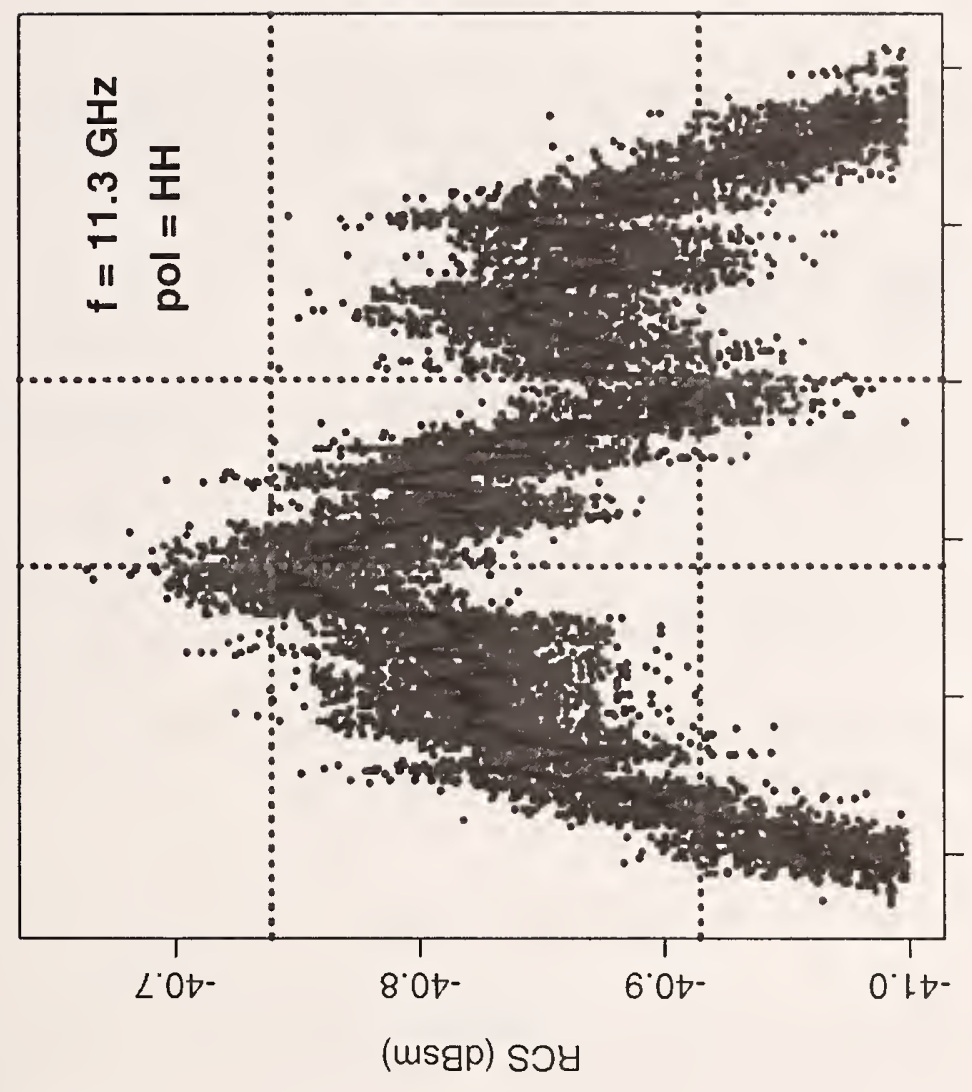

$\stackrel{\infty}{+}$

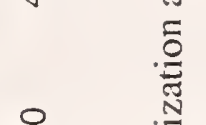

8

ํㅗㅇ

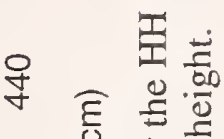

ह

- 志

ำ 을

8 을

흐

\&

흘.

뉴

马로

풍

可范

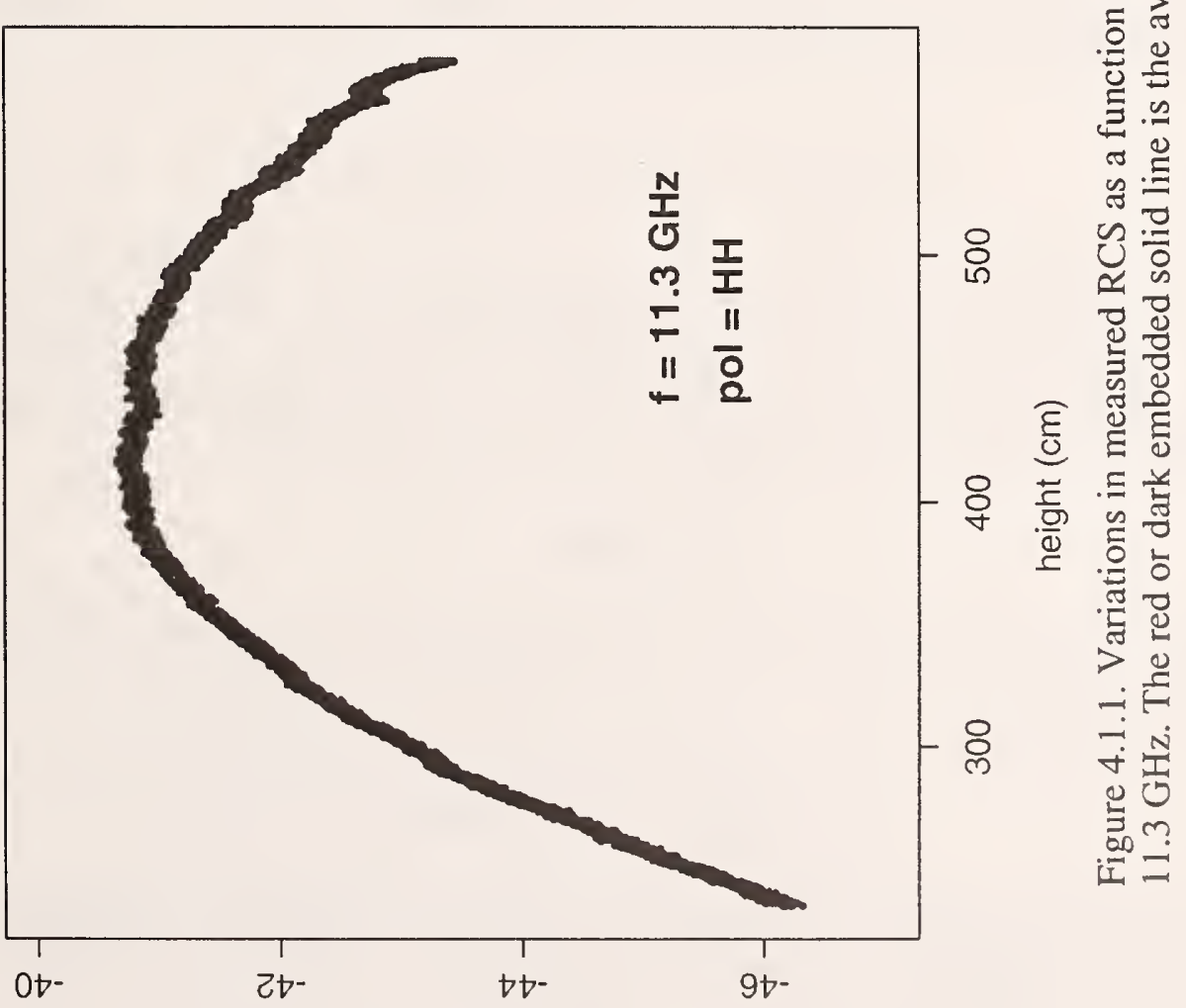

(usgp) soy 
receiving radar. Similarly, we have the reciprocal path $A_{R} T P A_{T}$. In general, all signal paths are of the form $A_{R}(T P)^{n} T^{k} A_{T}$ or $A_{R}(P T)^{n} P^{k} A_{T}$, where $n=1 . . \infty$ and $k=0$ or 1 . The interaction $I$ is then the sum of all signals, and can be represented as

$$
I=A_{R} \frac{(P T+P T P+T P+T P T)}{1 \pm T P} A_{T},
$$

where we choose the - sign if there is no phase change introduced in the signal paths. To estimate the target-mount interaction, we assume that

1. The monostatic calibration target and background measurements give the upper bounds of all possible bistatic RCS of the target and pylon, and

2. The second and fourth terms in the interaction I are an order of magnitude smaller than the first and third terms, and can therefore be neglected.

Then

$$
|I| \approx\left|A_{R}(P T+T P) A_{T}\right| \leq \alpha\left|A_{R}\left(P A_{T} A_{R} T+T A_{T} A_{R} P\right) A_{T}\right|=2 \alpha\left|A_{R} P A_{T}\right|\left|A_{R} T A_{T}\right|,
$$

where $\alpha$ is a scalar chosen to satisfy the above expression. If assumption 1 above holds, then $\alpha \approx 1$ gives the upper bound for the target-mount interaction.

Since the received signal is $S \equiv A_{R} T A_{T}$ and the background signal is $B \equiv A_{R} P A_{T}$, the dimensionaless interaction-to-signal ratio $\epsilon_{I}$ is

$$
\epsilon_{I} \equiv\left|\frac{I}{S}\right|=2 \alpha\left|A_{R} P A_{T}\right| \equiv 2 \alpha|B| .
$$

Equation (4.2.1) defines the dimensions of $\alpha$. Hence, we can use the background (clutter) RCS data (see Figures 4.2.1 and 4.2.2) to estimate this interaction. We could use a geometrical argument to estimate $\alpha$ as the weighted ratio of the curved cylindrical surface to the total surface area of the cylinder. However, the weighting constant is difficult to estimate using basic considerations. Instead, we use the upper bound $\alpha=4.5$.

$$
\left|\epsilon_{I}\right|=9|B| \text {. }
$$

Thus, the upper bound of the target-mount interaction is estimated to be $19 \mathrm{~dB}$ above the background measurement.

If we specify $\epsilon_{I}$ in decibels, then the target-mount uncertainty is given by

$$
\Delta \sigma_{d B}=-20 \log \left(1-10^{-\epsilon_{I} / 20}\right) .
$$

From Figures 4.2.1 and 4.2.2 we see that the background signal $|B|^{2} \approx-50 \mathrm{dBsm}$ for the vertical polarization; $\epsilon_{I}^{2}$ is then estimated to be $-31 \mathrm{~dB}$.

Estimated uncertainty: $\Delta \sigma(\mathrm{dB})=0.25$ 


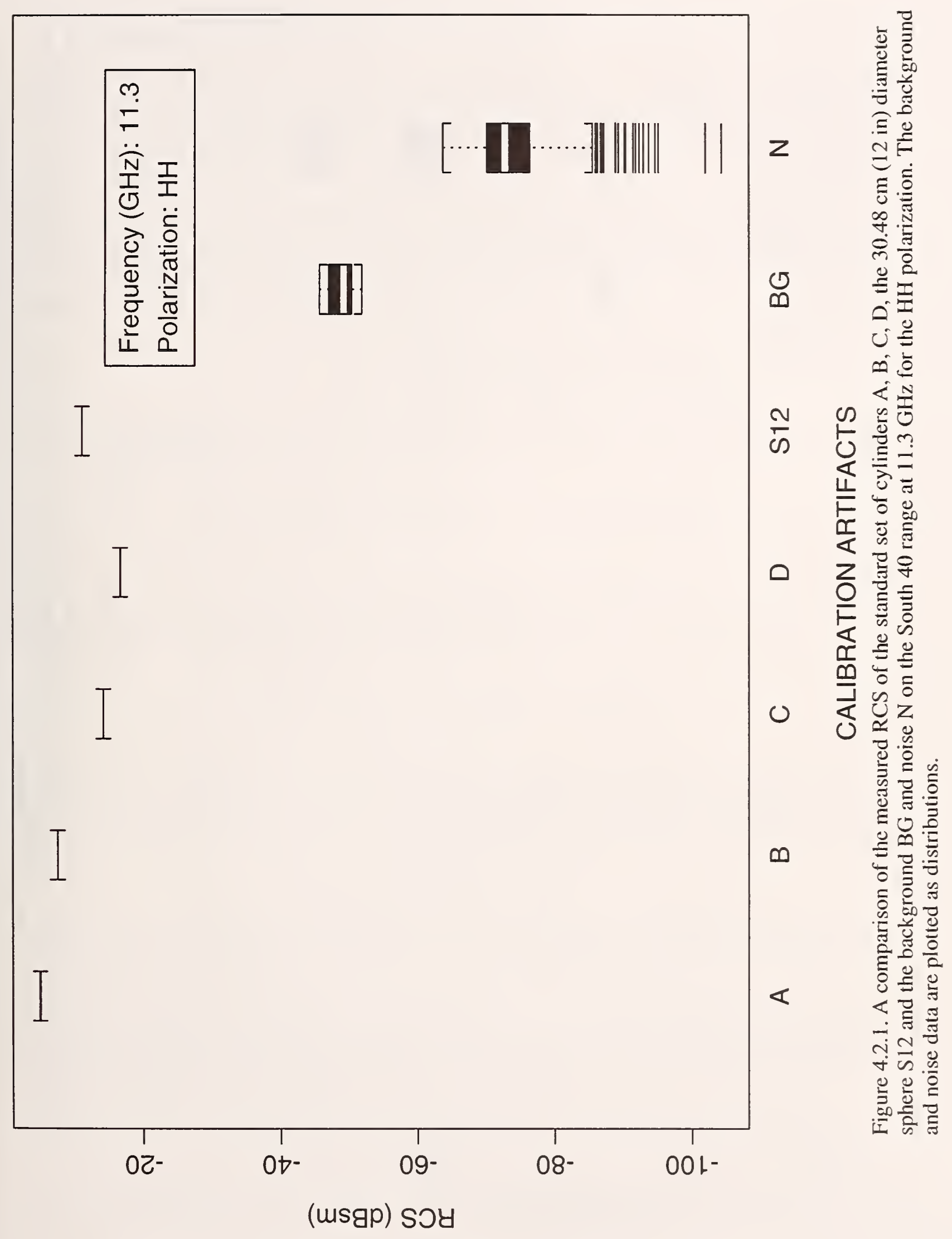




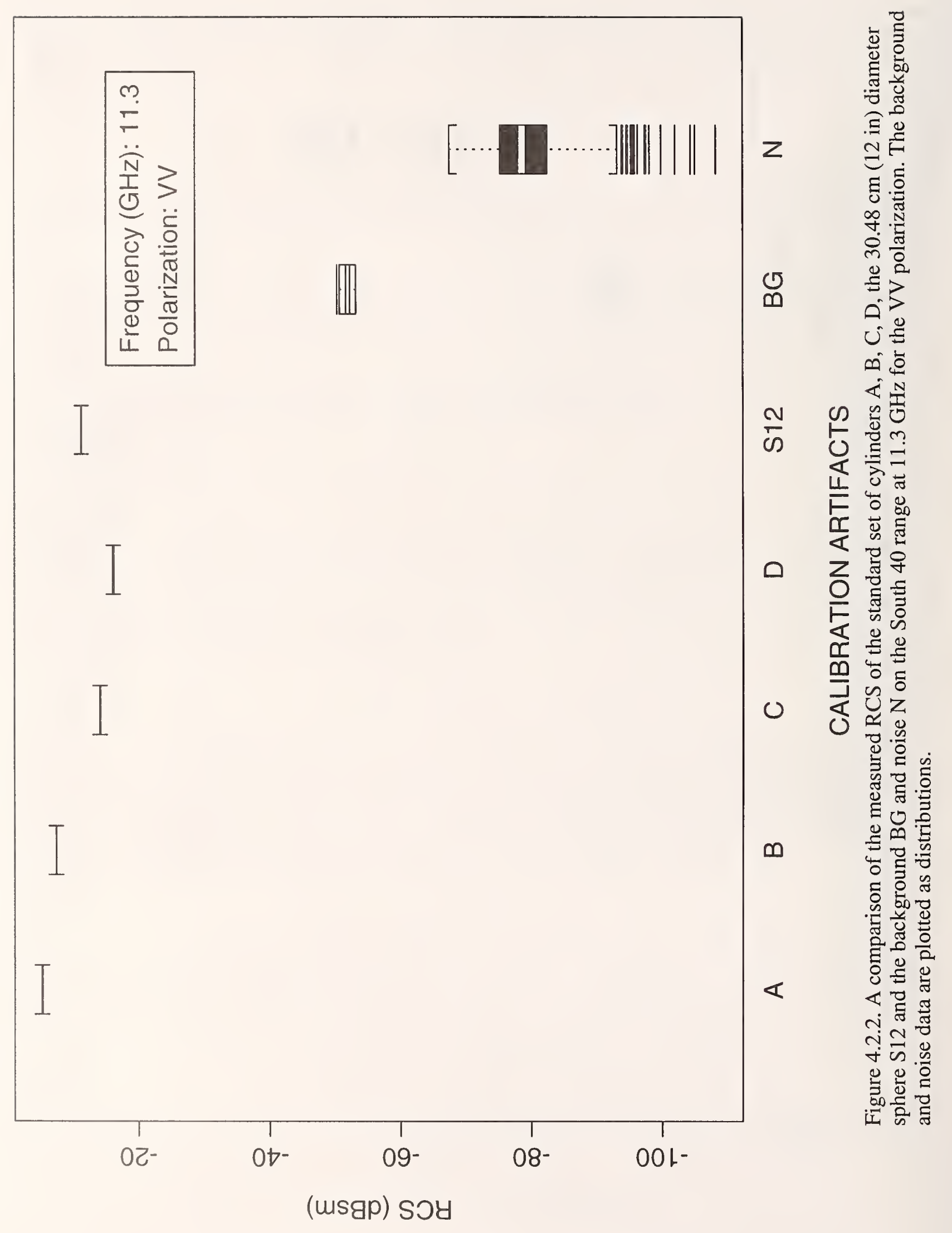




\subsection{CROSS POLARIZATION}

Here we account for the fact that, due to misalignment or physical imperfection, the antenna will not transmit or receive perfectly polarized ( $h$ or $v$ ) signals. However, even if the antennas have good polarization isolation, we can experience significant error in the co-polar RCS if the target's cross-polar $h v$ or $v h$ response is large compared to the co-polar $h h$ or $v v$ response.

We can fully correct for polarization errors if complete polarimetric calibration and measurements are performed [6-8]. Most RCS ranges do have the capability to perform polarimetric calibration and measurements, but only a few test programs require this.

We define the scattering matrix parameters:

$$
\begin{array}{ll}
r_{\mu \nu} & \text { antenna receiving coefficients, } \\
t_{\mu \nu} & \text { antenna transmitting coefficients, } \\
s_{\nu \mu} & \text { target scattering-matrix elements, }
\end{array}
$$

where $\nu$ and $\mu$ are either $v$ or $h$ for vertical or horizontal polarization, respectively. The received signal (when transmitting vertically) is [6-8]

$$
S_{v v}=r_{v v} s_{v v} t_{v v}+r_{v v} s_{v h} t_{h v}+r_{v h} s_{h v} t_{v v}+r_{v h} s_{h h} t_{h v}
$$

or

$$
S_{v v}=r_{v v} s_{v v} r_{v v}+2 r_{v v} s_{v h} r_{v h}+r_{v h} s_{h h} r_{h v}
$$

for a reciprocal [2] monostatic measurement system. We rewrite eq (4.3.1) as

$$
S_{v v}=r_{v v} s_{v v} r_{v v}\left(1+2 \frac{s_{v h}}{s_{v v}} \frac{r_{v h}}{r_{v v}}+\frac{s_{h h}}{s_{v v}} \frac{r_{v h}^{2}}{r_{v v}^{2}}\right) .
$$

The first term on the right of eqs (4.3.1) or (4.3.2) is the received copolar signal $S_{v v}^{(0)}$; the second $(v h)$ and third $(h h)$ terms are the cross-polarization signals. From eq (4.3.2) the cross-polarization terms are of first and second order in the antenna polarization-isolation ratio

$$
\rho_{v h} \equiv\left|\frac{r_{v h}}{r_{v v}}\right|<<1,
$$

when the antenna is in the vertical polarization state. When we interchange the indices $h$ and $v$, we obtain the expression valid for the horizontal polarization state. In general, we expect $\rho_{v h}$ to be small.

Equation (4.3.2) shows that the cross-polarization error is target dependent; the equation has three ratio parameters that determine the magnitude of the cross-polarization error. The ratio of the cross-polarization error terms in eq (4.3.2) is

$$
\frac{1}{2} \frac{s_{h h}}{s_{v h}} \frac{r_{v h}}{r_{v v}}
$$

which is not necessarily small, even if the antenna polarization isolation is small. 


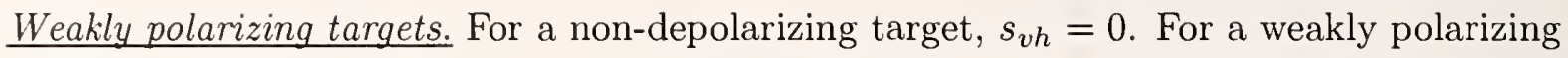
target

$$
s_{v h} \approx 0 .
$$

If $s_{h h}>>s_{v h}$, the error is of second order in $\rho_{v h}$, but could still be large if $s_{h h} / s_{v v}>>1$. Strongly depolarizing target. By definition, we have strongly depolarizing targets when the ratio of the two cross-polarization terms in eq (4.3.2) satisfies

$$
\frac{1}{2}\left|\frac{s_{h h}}{s_{v h}} \frac{r_{v h}}{r_{v v}}\right| \leq 1 .
$$

Eq (4.3.2) can in general be written as

$$
S_{v v}=r_{v v} s_{v v} r_{v v}\left(1+2 \alpha \frac{s_{v h}}{s_{v v}} \frac{r_{v h}}{r_{v v}}\right),
$$

where $\alpha$ is some complex constant. When $\alpha=2$ the two error terms in eq (4.3.2) are equal, and when $\alpha=1$ the last term in eq (4.3.2) has been neglected (strong depolarization). The error now is of first order in $\rho_{v h}$, but its magnitude still depends on the ratio of the target's crosspolar $s_{v h}$ and copolar $s_{v v}$ scattering characteristics.

As an example, we assume a strongly depolarizing unknown target with $\alpha=2$ and $s_{v v}=$ $s_{v h}$, so that the error signal is

$$
\left|\frac{\Delta S}{S_{v v}^{(0)}}\right|=4\left|\frac{r_{v h}}{r_{v v}}\right|
$$

where $\Delta S \equiv S_{v v}-S_{v v}^{(0)}$. Thus

$$
\Delta \sigma(\mathrm{dB})=-20 \log \left(1-4 \times 10^{-\epsilon_{p} / 20}\right),
$$

where $\epsilon_{p}=20 \log \left(r_{v h} / r_{v v}\right)$ is the antenna polarization isolation. If the polarization isolation is $\epsilon_{p}=30 \mathrm{~dB}$, then the cross-polarization component uncertainty is $1.2 \mathrm{~dB}$.

Estimated uncertainty: $\Delta \sigma(\mathrm{dB})=1.20$ (strong depolarization)

Estimated uncertainty: $\Delta \sigma(\mathrm{dB})=0.30$ (weak depolarization, by assumption)

\subsection{DRIFT}

All radar systems are subject to drift due to variations in environmental conditions. On outdoor ranges, changes in temperature can cause signal variations; similarly, wind and humidity can also influence the results of measurements. To study system drift we need to observe and characterize (i) short-term, (ii) intermediate-term, and (iii) long-term drifts in the received signal. Simple analysis of drift data should enable us to determine the corresponding calibration and measurement uncertainties. We define short-term drift as the variation in the received signal during a calibration interval; in this study the calibration interval was $20 \mathrm{~s}$, and we used the standard cylinder set to make drift measurements. Simultaneous recordings of temperature, wind velocity and humidity are highly desirable. 
The corresponding uncertainty can be used in the Calibration Target Uncertainties table (see Table 2.1).

We define intermediate-term drift as the variation in the received signal during a measurement interval. In practice, this is the interval between pre-calibration and post-calibration measurements that bracket the measurements on a test target. This could be between 2 and 12 hours, and will depend on the details of the test plan. Simultaneous recordings of temperature, wind velocity and humidity are highly desirable. The corresponding uncertainty can be used in the Test Target Uncertainties table (see Table 2.1).

We define long-term drift as the drift observed during an extended period, for example, 12 to 24 hours. Such observations are used to monitor system performance and stability, but are not used to determine system uncertainties. During such measurements the temperature, wind conditions and humidity, as functions of time, should also be recorded. A historical data base can help identify unexpectedly large variations during measurements of long-term drift. Such large variations must be understood, and, if necessary, corrective measures should be taken to improve overall system stability.

We now describe how we determined the (i) short-term, (ii) intermediate-term and (iii) long-term drifts.

Short-term drift. We used the standard calibration cylinders to determine the shortterm drift of the radar system. The corresponding uncertainty is used in the Calibration Target Uncertainty table. Each of the four cylinders was measured for $20 \mathrm{~s}$. The variations in the received signals are shown in Figures 4.4.1 and 4.4.2. We define drift during the measurements on a particular cylinder as the range of the received signals expressed in decibels. The system short-term drift is then defined as the maximum range observed during the measurements on the four cylinders. We observed drifts of approximately 0.10 , $0.06,0.10$, and $0.08 \mathrm{~dB}$ for the $\mathrm{HH}$ polarization, and $0.10,0.06,0.06$ and $0.15 \mathrm{~dB}$ for the VV polarization for cylinders $\mathrm{A}, \mathrm{B}, \mathrm{C}$, and $\mathrm{D}$, respectively. Then, by definition the calibration or short-term drift at $11.3 \mathrm{GHz}$ is observed to be $0.15 \mathrm{~dB}$. This observation is valid at signal levels of -5 to $-17 \mathrm{dBsm}$, the RCS range of the four cylinders.

Additionally, in Figure 4.4.3 we show the drift during measurements made on a sphere of $30.48 \mathrm{~cm}$ (12 in) diameter. We observe the drift to be $<0.15 \mathrm{~dB}$, which is the drift calibration uncertainty as determined above.

Intermediate-term drift. The overall configuration of the components of the RCS measurement system is outlined in Appendix B. To assess the cause of intermediate-term drift in the overall RCS measurement system, three different test configurations were used. The first tested the stability of the radar electronics in the radar room, which are common to all of the operational bands of the radar. The second configuration added the S, C, X and $\mathrm{Ku}$ Hoffman boxes to the radar electronics included in the first test. The third test had the radar configured for RCS measurements and used cylinder A on a foam column as the target. The system was not calibrated for this stability test. Because of limitations in the data acquisition software we were limited to observation times of 10,000 seconds or less. Typically, we observed the system drift for about 8,000 seconds. Longer-term observations will be made once the data acquisition software has been updated. 


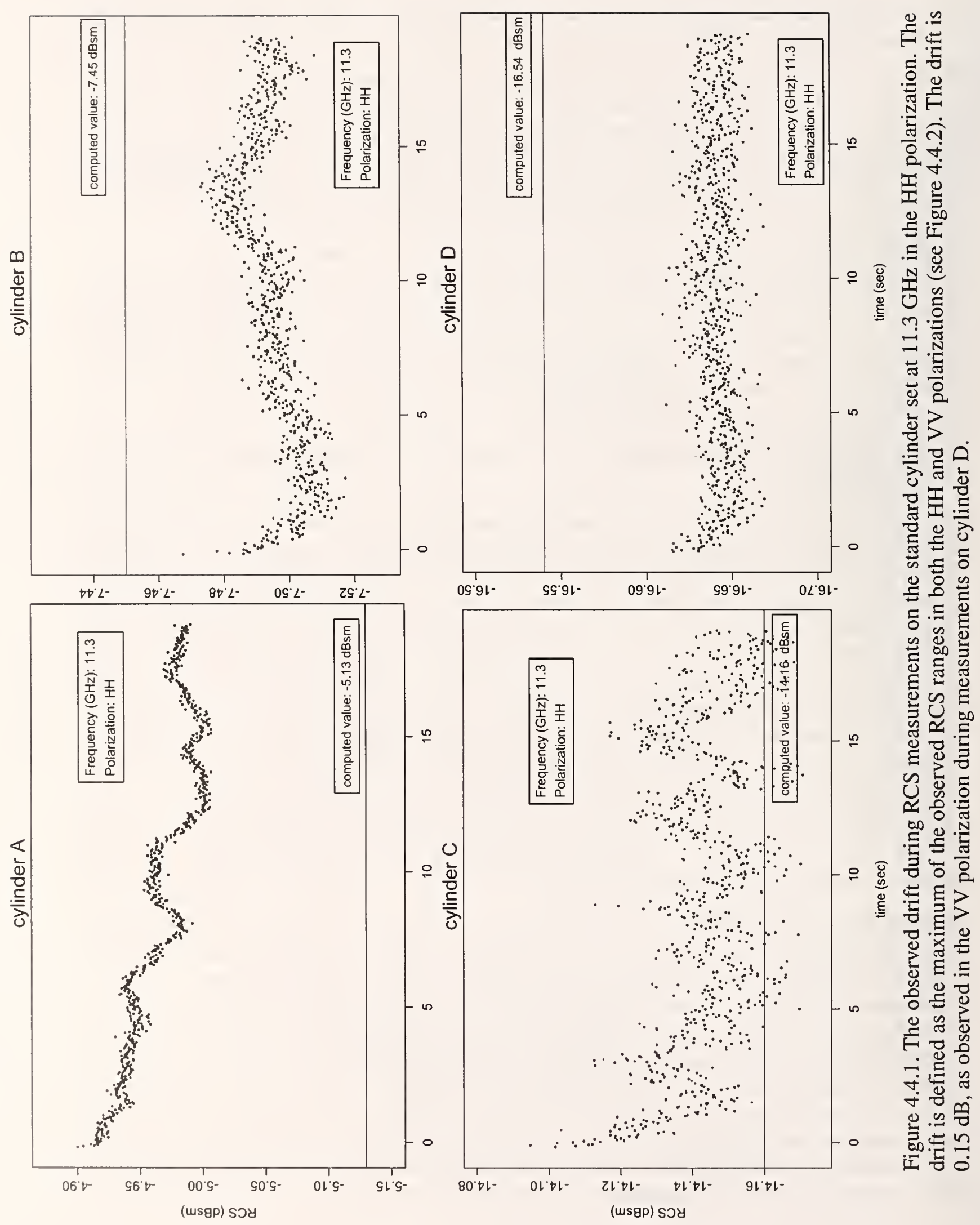



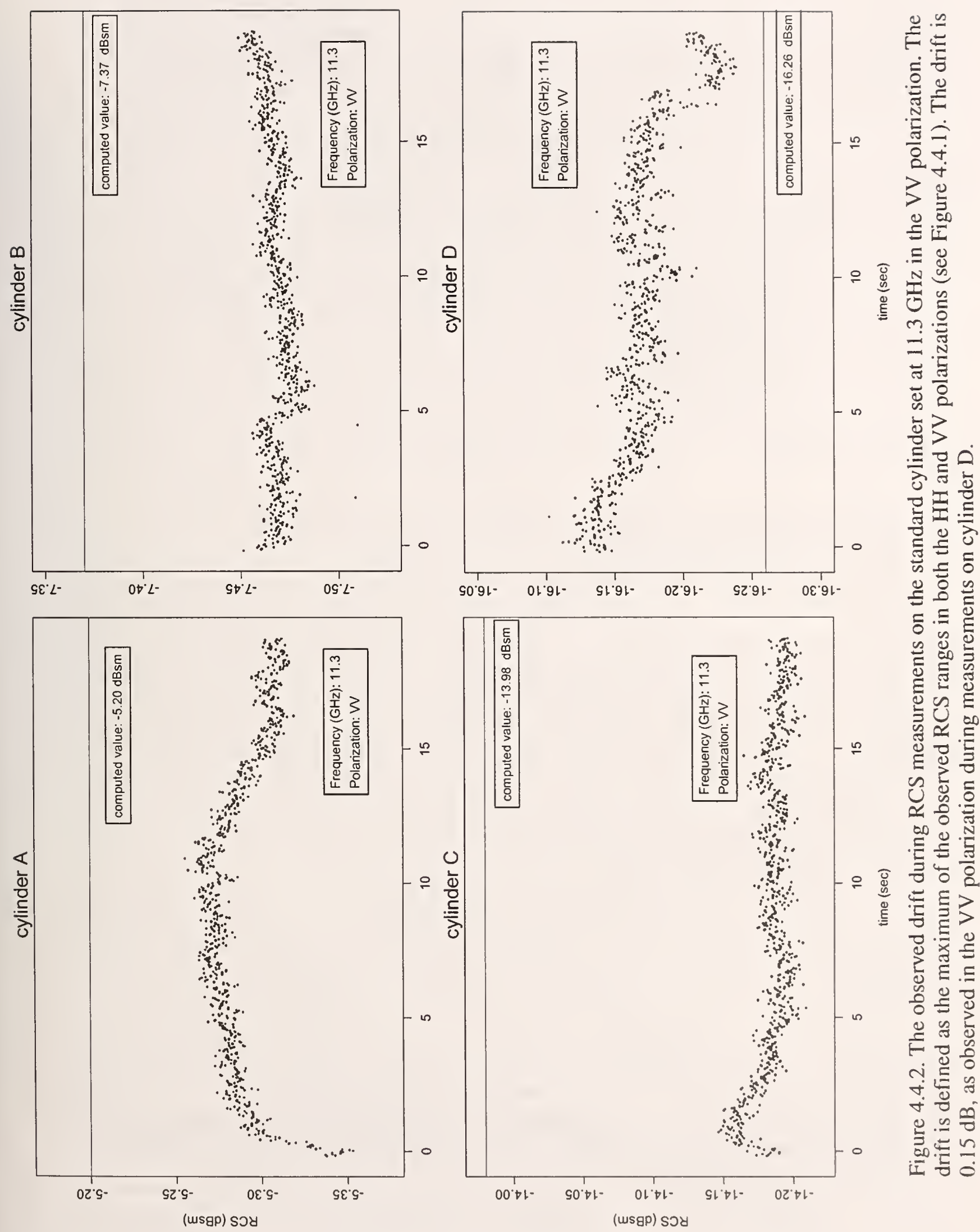

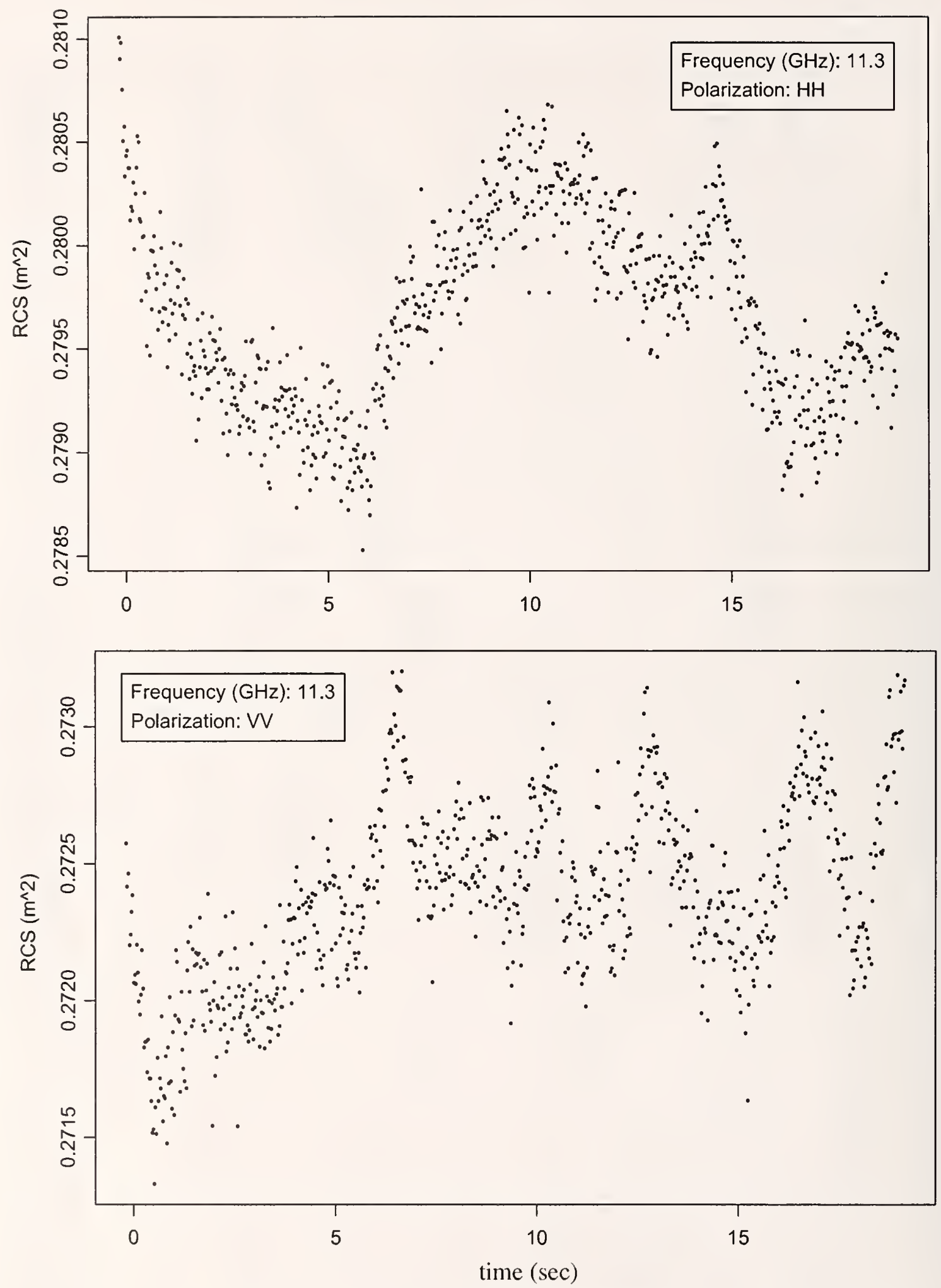

Figure 4.4.3. The observed drift during RCS measurements at $11.3 \mathrm{GHz}$ on a $30.48 \mathrm{~cm}$ (12 in) diameter sphere. The drift, defined as the maximum range in the observed data, is determined to be $<0.15 \mathrm{~dB}$. 
The first test measured the system drift attributable to the electronics located in the radar room. For this test the $2 \mathrm{GHz}$ IF transmit pulse was looped back into the $2 \mathrm{GHz}$ IF receive input of the $\mathrm{HH}$ and VV channels through an attenuator. No unusual sources of drift were noted in this testing, but the testing did allow fine tuning of the cycling of the radar room air conditioning system to minimize the drift in the radar room electronics.

The second test added the $\mathrm{S}, \mathrm{C}, \mathrm{X}$, and $\mathrm{Ku}$ band $\mathrm{rf}$ components in the $\mathrm{S}, \mathrm{C}, \mathrm{X}$, and $\mathrm{Ku}$ Hoffman boxes (located close to the associated antennas) to the electronics used in the first test. This was a closed-loop test: the $\mathrm{HH}$ and VV rf transmitter pulses were looped back into the respective receiver inputs through rf pads chosen to set the signal level in each of the four Hoffman boxes. Observations were made at $3.57 .1,9.3$, and $16.5 \mathrm{GHz}$, which are the center frequencies of the high $\mathrm{S}$, high $\mathrm{C}$, low $\mathrm{X}$ and high $\mathrm{Ku}$ sub-bands, respectively. Both the $\mathrm{HH}$ and $\mathrm{VV}$ polarizations at all four frequencies were observed simultaneously using an eight-element waveform. The measurements were made at night to duplicate typical test conditions. The maximum drift observed at $9.3 \mathrm{GHz}$ was 0.1 $\mathrm{dB}$ for the VV polarization (see Figure 4.4.4), and was $0.2 \mathrm{~dB}$ at $16.5 \mathrm{GHz}$ for the $\mathrm{HH}$ polarization. Improved temperature stability of the Hoffman boxes would likely reduce this source of drift.

For the third test the system was in its operational configuration, with the 900 calibration cylinder (see Figure 4.1 and Table 4.1) mounted on a foam column. Simultaneous drift measurements at the four frequencies for both the $\mathrm{HH}$ and VV polarizations were made with the eight-element waveform used in the second test.

Full system-drift measurements were made by use of a 900 calibration cylinder (see Figure 4.1 and Table 4.1) as the target. The same eight-element waveform (see above) was used to simultaneously observe the system drift at the center frequencies of the four bands for both polarizations. Again these measurements were made at night to duplicate typical test conditions. During the night-time measurements the temperatures were cold enough that attention was given to adequate heating of the Hoffman boxes to control signal drift originating in the Hoffman boxes. The $\mathrm{S}$ band Hoffman box had four transmitters that could be put in standby mode to provide the required heat load. The other Hoffman boxes had only two transmitters; the $\mathrm{C}$ band appeared to be more sensitive to temperature than the $\mathrm{X}$ or $\mathrm{Ku}$ bands, so an available floor heater was used to deliver additional heating to the C-band Hoffman box. A maximum drift of $0.2 \mathrm{~dB}$ was observed at $16.5 \mathrm{GHz}$ for the $\mathrm{HH}$ polarization. We observed drift at $9.3 \mathrm{GHz}$ but not at $11.3 \mathrm{GHz}$; the observed drift was $0.3 \mathrm{~dB}$ for both polarizations, as shown in Figure 4.4.5. We can assume that this is the test target uncertainty for all of $\mathrm{X}$ band, since only the limiters change when switching frequencies within a band.

Long-term drift. We define long-term drift as drift occuring in a period of 12 to 24 hours. Currently the data-collecting algorithm does not allow us to collect data for longer than 10,000 seconds. A new algorithm is being implemented to eliminate this limitation. We will study the long-term system drift in detail after this improvement is operational.

Estimated uncertainty: $\Delta \sigma(\mathrm{dB})=0.15$ for short-term drift (calibration)

Estimated uncertainty: $\Delta \sigma(\mathrm{dB})=0.30$ for intermediate-term drift (target) 

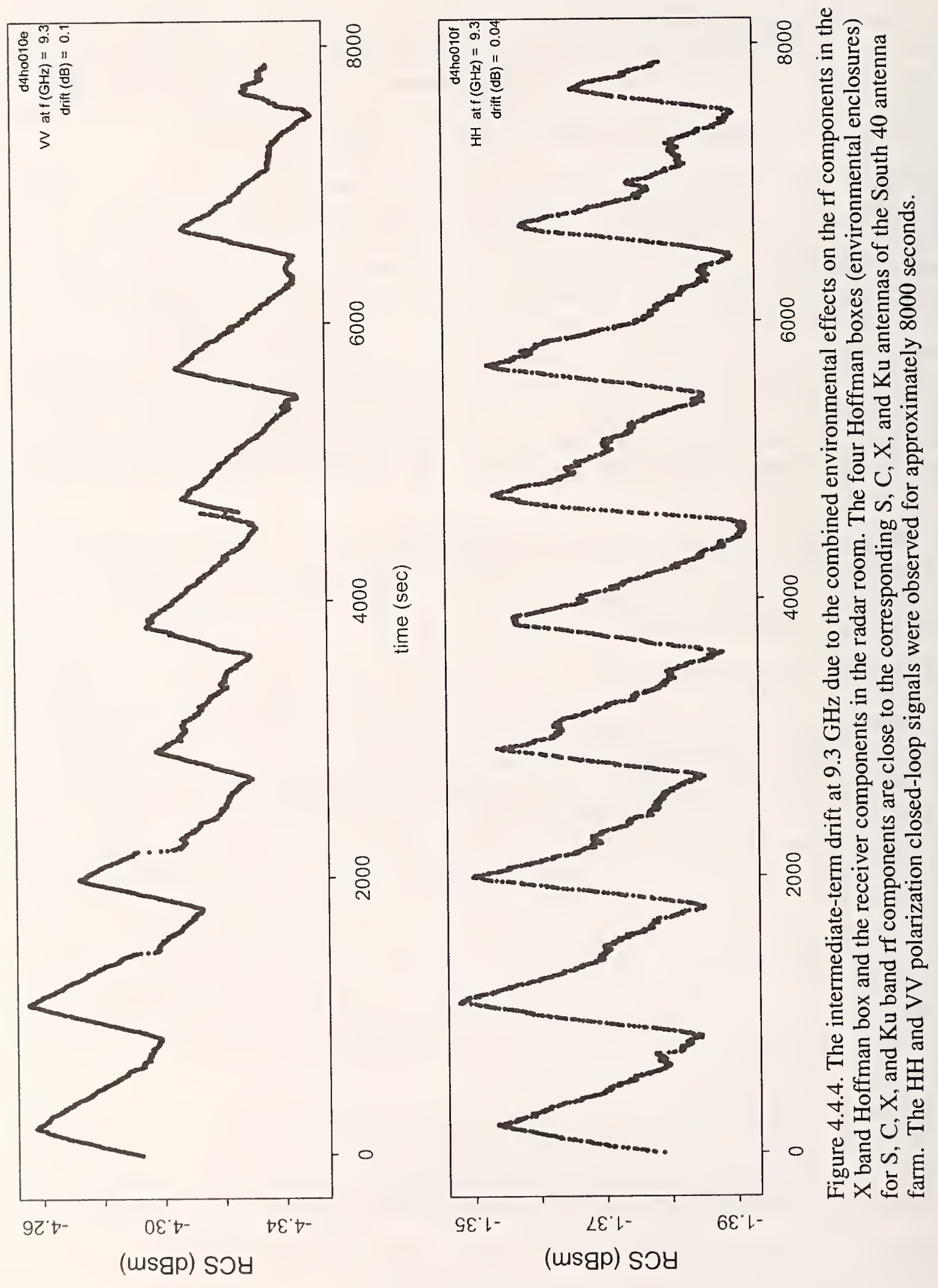

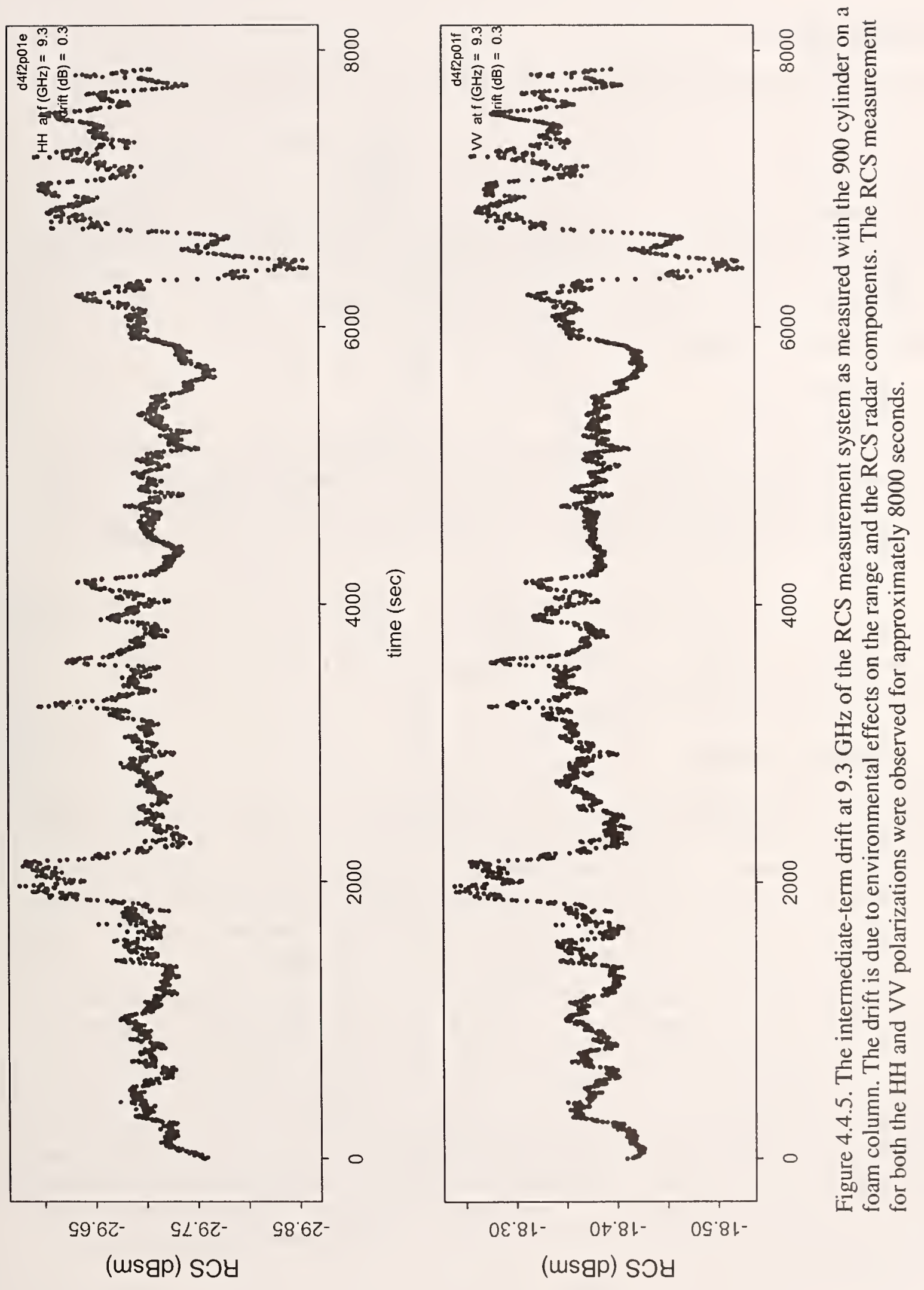


\subsection{FREQUENCY}

From eq (7a) the component of uncertainty in RCS due to uncertainty in frequency is

$$
\Delta \sigma(\mathrm{dB})=-20 \log \left(1-\frac{\Delta f}{f}\right) .
$$

The uncertainty $\Delta f$ in frequency may be taken as the effective bandwidth of the system; that is, as the minimum of the transmitted bandwidth and the received bandwidth. This assumes that the bandwidth is not affected by digital filtering in software gating. At $f=9.3 \mathrm{GHz}$, we use $\Delta f=200 \mathrm{MHz}$ to obtain the uncertainty.

Estimated uncertainty $\Delta \sigma(\mathrm{dB})=0.16$

\subsection{INTEGRATION}

Integration uncertainty is due to target motion occuring during a number of pulses that are integrated within the hardware and/or software. Ideally this motion is entirely negligible, but in windy conditions the calibration might move by a significant fraction of a wavelength. The uncertainty due to the uncertainty in position $\Delta R$ during the pulse integrations is

$$
\Delta \sigma(\mathrm{dB})=-40 \log \left(1-\frac{\Delta R}{R}\right)
$$

Most likely this is negligible, since $R$ is very large compared to the wavelength.

Estimated uncertainty $\Delta \sigma(\mathrm{dB})=0$

\subsection{I-Q IMBALANCE}

If there is an I-Q imbalance, the measured amplitude will be a function of input phase. I-Q imbalance was measured with the radar by feeding the transmitted IF pulse at $2 \mathrm{GHz}$ through a step attenuator and a manual phase shifter back to the IF $\mathrm{H}$ or V input channel. Data were obtained for a number of coherent integrations (NCI) (within the hardware) and attenuation levels as a function of phase. Specifically, NCI was set to 2, 4, 8, 16, and 32 and the attenuation levels were set at 0 to $50 \mathrm{~dB}$ in $10 \mathrm{~dB}$ steps.

The orthogonal-distance regression (ODR) technique [1-3] was used to determine the I-Q circle that best fits the measurements. The ODR parameters provide the DC offset and the uncertainty in the radius of the IQ circle. The total measurement uncertainty is then defined as the RSS of the DC offset and the radial uncertainty.

In Figure 4.7.1 we show the H-channel I-Q circularity data and the I-Q circles as determined by the least-squares analysis using ODRPACK [12] for NCI of 2 and 8 pulses and attenuation levels of $0 \mathrm{~dB}$ and $50 \mathrm{~dB}$. The + at the center of the circle indicates the DC offset of the measurement system. The uncertainty in the radius of the circle is automatically determined from the data by ODRPACK. The uncertainties were determined by use of the expressions

$$
\Delta_{I Q}^{2}=I_{0}^{2}+Q_{0}^{2}+\delta r^{2},
$$



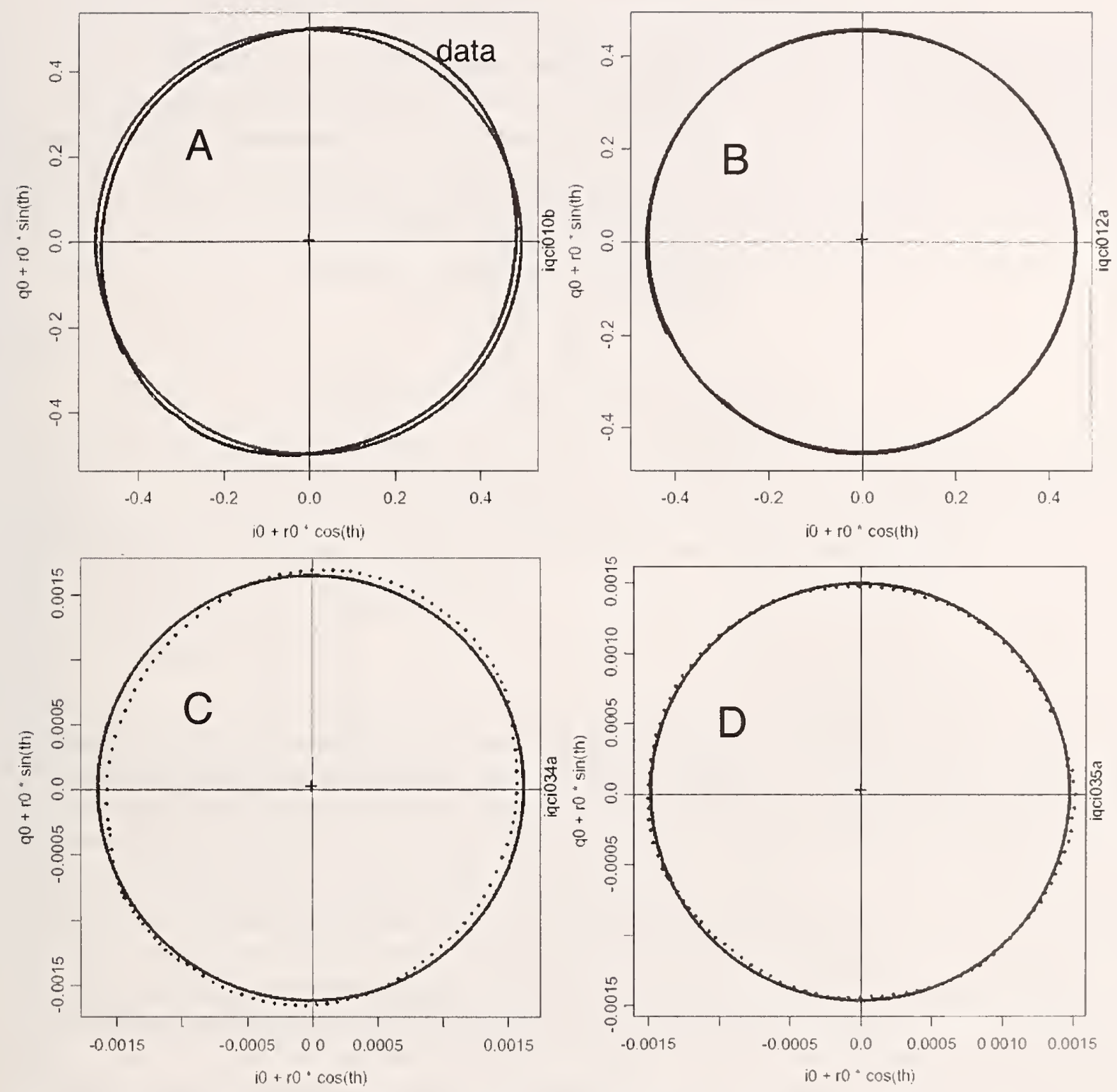

Figure 4.7.1. The I-Q circularity as a function of attenuation and NCI. (A) $0 \mathrm{~dB}$ attenuation and $\mathrm{NCI}=2$; (B) $0 \mathrm{~dB}$ attenuation and $\mathrm{NCI}=8$; (C) $50 \mathrm{~dB}$ attenuation and $\mathrm{NCI}=2$; (D) $50 \mathrm{~dB}$ attenuation and $\mathrm{NCI}=8$. The data are indicated in blue (dotted), and the IQ circles obtained using ODRPACK are drawn in red (solid). The + signs at $(\mathrm{I0}, \mathrm{Q} 0)$ indicate the centers of the circles, which give the DC offsets. The total measurement uncertainty is defined as the RSS of the DC offset and the uncertainty in the radius $\mathrm{r} 0$ of the IQ circle. 
where $\left(I_{0}, Q_{0}\right)$ is the complex DC offset, and $\delta r$ is the uncertainty in the radius $r_{0}$ of the $\mathrm{I}-\mathrm{Q}$ circle, and

$$
\Delta \sigma_{d B}=20 \log \left(1-\Delta_{I Q} / r_{0}\right) .
$$

The total I-Q uncertainties for each of the measurements in Figure 4.7.1 are given in Table 4.7.1. We see that the I-Q uncertainty is not a sensitive function of the NCI, but is somewhat more strongly dependent on the attenuation level. We choose the maximum uncertainty (rounded to the nearest 10th of a dB) in Table 4.7.1 as the system I-Q uncertainty.

Table 4.7.1. The I-Q uncertainty as a function of attenuation and NCI.

\begin{tabular}{cccc}
\hline \hline$\underline{\mathrm{dB} / \mathrm{NCI}}$ & $\underline{2}$ & $\underline{8}$ \\
0 & 0.04 & 0.04 \\
50 & 0.11 & 0.10 \\
\hline
\end{tabular}

Estimated uncertainty: $\Delta \sigma(\mathrm{dB})=0.10$

\subsection{NEAR FIELD}

The small calibration cylinder is in the far-field of the antenna. Therefore, the near-field uncertainty is negligible.

Estimated uncertainty: $\Delta \sigma(\mathrm{dB})=0$

\subsection{NOISE - BACKGROUND}

In Figures 4.2.1 and 4.2.2 we show the RCS measurements of the system noise, the background and clutter, and the calibration cylinders. The cylinders and the background and clutter are measured coherently, but the system noise is measured incoherently. Both the background and system noise signals are significantly lower than the RCS of the cylinders.

System Noise. We first calibrate the radar system, and then record the RCS of the system noise after the bracket pulse has been disabled from the radar transmitter to suppress rf transmission. This is accomplished either by disconnecting the bracket cable going to the transmitter or by turning off the bracket pulse in the collection computer. System noise is measured for a predetermined period by use of a waveform identical to that used in the test target measurements.

From Figures 4.2.1 and 4.2.2 we find that the maximum $|N|^{2}=-70 \mathrm{dBsm}$, which occurs in the $\mathrm{HH}$ polarized noise measurements. We use this value to determine the system background-noise uncertainty.

Background Measurements. The background or clutter measurement is identical to a calibration or test-target measurement without the target. Thus, the reflected signal originates only from the environment and the pylon. We expect this signal to be substantially below the signal produced by the calibration and test targets, but it is usually higher than the system noise.

From Figures 4.2.1 and 4.2.2 we see that the maximum $|B|^{2}=-45 \mathrm{dBsm}$, which occurs 
for the $\mathrm{HH}$ polarized background measurement. We use this value to determine the system background-noise uncertainty.

The overall background-noise is the complex sum of $B$ and $N$, and

$$
|B+N|^{2} \approx|B|^{2}=-45 \mathrm{dBsm}
$$

since the noise $N$ is significantly less than the background (clutter) RCS.

The uncertainty in the system noise-background during calibration is determined using the theoretical RCS of cylinder $\mathrm{D}$, which is $-16.5 \mathrm{dBsm}$ for the $\mathrm{HH}$ polarization. Thus

$$
\epsilon \equiv|S / B|^{2}=28.50 \mathrm{~dB}
$$

and

$$
\Delta \sigma_{B N} \equiv-20 \log \left(1-10^{-\epsilon / 20}\right) \approx 0.35 \mathrm{~dB} \text {. }
$$

Obviously, the accuracy of RCS measurements would be notably improved if we could minimize the magnitude of noise-background uncertainty. Measurement techniques to minimize the contribution of the background (clutter) present on the range are under study [9-10].

Estimated uncertainty: $\Delta \sigma(\mathrm{dB})=0.35$

\subsection{NONLINEARITY}

The linearity measurements at S, C, X, and $\mathrm{Ku}$ bands were made using a closed-loop configuration in the environmental enclosures (Hoffman boxes). Each Hoffman box contains a rf converter, transmitters (nominally four each), and a switch pack. The switch pack contains the HH and VV limiters for the high and low sub-bands, sub-band switching for the high and low sub-bands, and rf step-attenuators and low-noise HH and VV preamplifiers. The Hoffman boxes are located close to the antennas.

The rf transmitter drive pulse from the converter was connected to the receiver input through a manually controlled HP step attenuator that had been calibrated at NIST. The two-section step attenuator consisted of an 11-step section with $1 \mathrm{~dB} / \mathrm{step}$ attenuation, and a 7-step section with $10 \mathrm{~dB} /$ step attenuation. The rf pads in series with the step attenuator provided the appropriate starting signal level for each linearity measurement. The step attenuator was located outside the Hoffman boxes to keep the boxes closed during the measurements. The desired attenuation was set manually at predetermined intervals measured with a stopwatch. The received signals as a function of attenuation levels were recorded for each frequency of interest.

In Figure 4.10.1 we show the attenuation data for $1 \mathrm{~dB}$ and $10 \mathrm{~dB}$ attenuation. The spurious signals present in the data when the attenuators were switched have been supressed. In Figure 4.10.2 we show the change in signal levels as the attenuation level is increased; in Figure 4.10 .3 we show the deviation of the change in signal levels from the nominal step values of $1 \mathrm{~dB}$ or $10 \mathrm{~dB}$. We see from these figures that system nonlinearity is very acceptable from 0 to $55 \mathrm{~dB}$ attenuation. Increased attenuation beyond this level, however, 

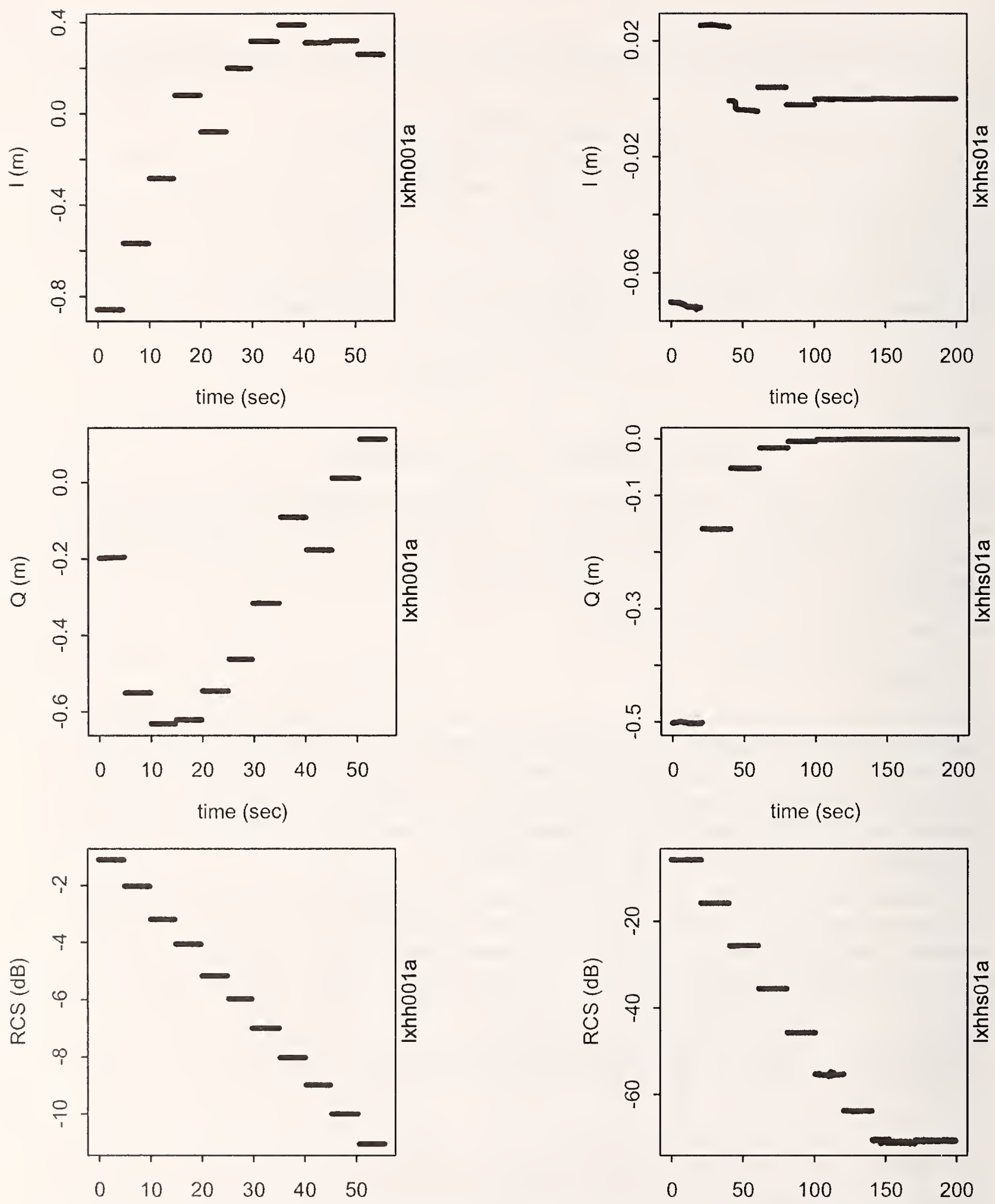

Figure 4.10.1. Nonlinearity measurements at $11.3 \mathrm{GHz}$ in $1 \mathrm{~dB}$ and $10 \mathrm{~dB}$ steps. The $\mathrm{I}$ and Q plots show the real-time data, and the RCS plots in $\mathrm{dB}$ are obtained from the sum of squares of $\mathrm{I}$ and $\mathrm{Q}$. The spurious data observed when switching attenuation levels have been suppressed. We observe significant nonlinearity only below $-60 \mathrm{~dB}$ (see Figure 4.10.2).

32 

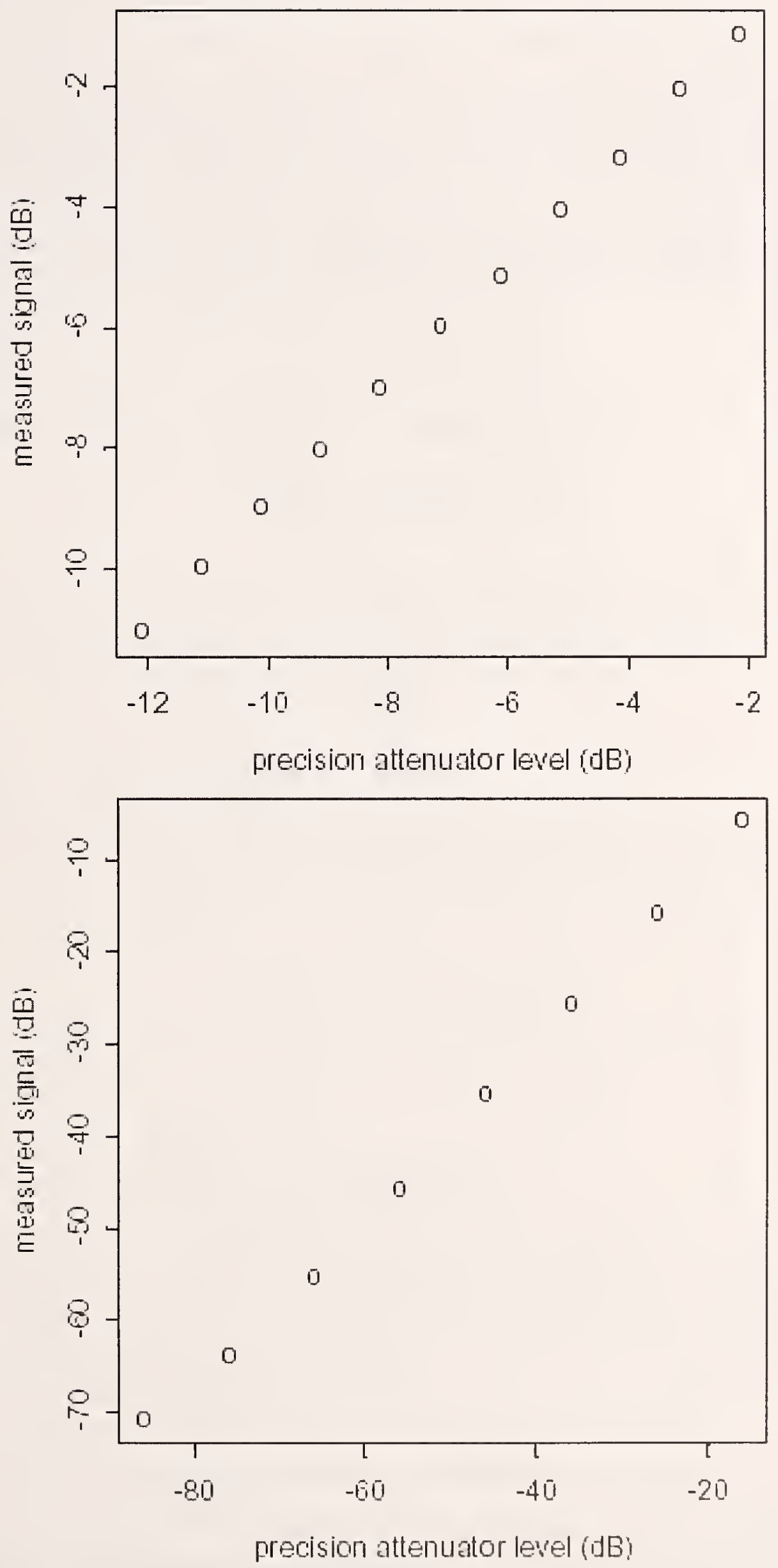

Figure 4.10.2. Measured signal as a function of precision attenuator levels in decibels at $11.3 \mathrm{GHz}$. 

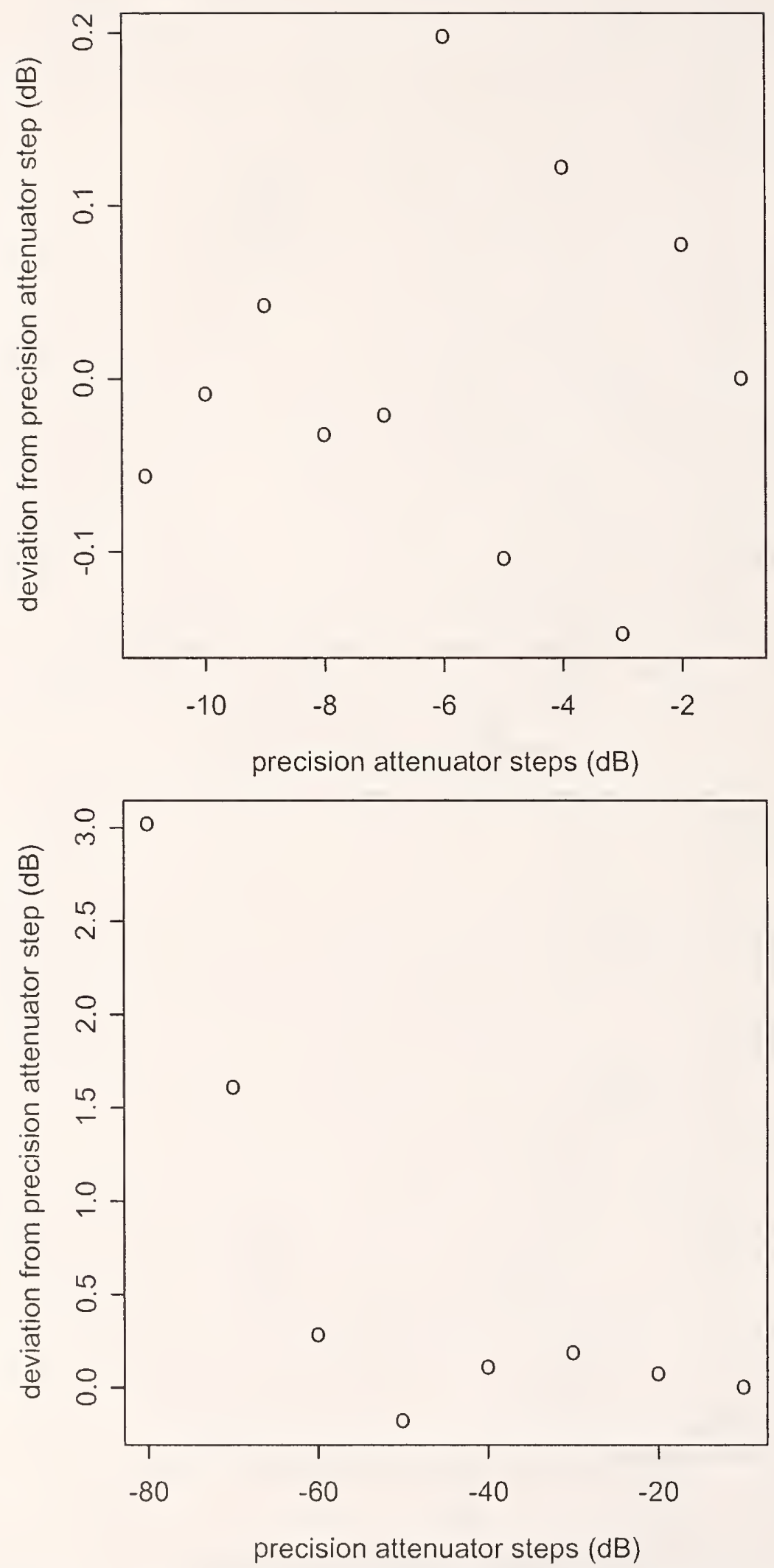

Figure 4.10.3. The deviation of the change in signal levels from the nominal step values of $1 \mathrm{~dB}$ (top) or $10 \mathrm{~dB}$ (bottom) at $11.3 \mathrm{GHz}$. The nonlinearity observed at greater than $60 \mathrm{~dB}$ attenuation is most likely due to of leakage. 
shows significant departures from linearity. The nonlinearity is more pronounced as the attenuation is increased. This is probably due to cable-to-cable rf signal coupling around the attenuator, but the cause has not been ascertained.

Based on the signal difference data in Figure 4.10.3 we determined the upper bound of the system nonlinearity uncertainty to be $0.25 \mathrm{~dB}$ for signal levels between 0 and $-60 \mathrm{~dB}$.

Estimated uncertainty $\Delta \sigma(\mathrm{dB})=0.25$

\subsection{RANGE}

The uncertainty in RCS due to uncertainty in range $\mathrm{R}$ is given by [1]

$$
\Delta \sigma(\mathrm{dB})=-40 \log \left(1-\frac{\Delta R}{R}\right),
$$

where the error in the range $\Delta R$ is taken to be some fraction of the calibration target dimension along the radar-to-target line of sight. From Tables 4.1 and B.2 (see Appendix B) we see that the fractional error in eq (4.11.1) is very small. Hence, the uncertainty in RCS due to range error is negligible, that is, less than $0.01 \mathrm{~dB}$.

Estimated uncertainty $\Delta \sigma(\mathrm{dB})=0$

\subsection{TARGET ORIENTATION}

The error in orientation of a calibration target can lead to large errors in the measured RCS of the target (relative to the RCS computed for the orientation assumed in the modeling). We can use computer simulations to determine the RCS as a function of orientation. The uncertainty is given by

$$
\Delta \sigma(\mathrm{dB})=-10 \log \left(1-\frac{\partial \sigma}{\partial \theta} \frac{\Delta \theta}{\sigma}\right) .
$$

We have not performed the required computer simulations, but we will assume here an uncertainty of $0.1 \mathrm{~dB}$.

Estimated uncertainty $\Delta \sigma(\mathrm{dB})=0.1$

\subsection{REFERENCE RCS (COMPUTATION)}

The uncertainty in the reference RCS, which is obtained computationally, is due to two effects: (1) the computer codes do not compute exact theoretical values, (that is, there is a computational error), and (2) the actual physical calibration artifact has physical imperfections, whereas the computer model of the ideal shape of the artifact is exact. The generally accepted uncertainty here is $0.1 \mathrm{~dB}$.

Estimated uncertainty $\Delta \sigma(\mathrm{dB})=0.1$

\subsection{COMBINED CALIBRATION UNCERTAINTY (RSS)}

Here we obtain the RSS of the component uncertainties in sections 4.1 through 4.13 (see eq (5), Section 2).

Estimated uncertainty $\Delta \sigma(\mathrm{dB})=0.71$ 


\section{CONCLUSION AND SUMMARY}

We have presented a measurement-based uncertainty analysis of the calibration of the RCS measurement system at EVR, NAVAIR at China Lake, CA. All sources of uncertainy were examined and an overall calibration uncertainty was obtained at $11.3 \mathrm{GHz}$. All assumptions were clearly identified and stated. The worst-case for each component uncertainty was obtained so that no assumptions about statistical distributions needed to be made. The overall uncertainty was obtained by the use of RSS. The procedure followed in this study can be easily repeated at any frequency of interest.

Acknowledgement. This work was supported by the DoD triservice Calibration Coordination Group (CCG). Special thanks are extended to H. Glick, M. Cunevalis, and R. Cleland of the Microwave Subgroub for their long-term support. 


\section{REFERENCES}

[1] R. C. Wittmann, M. H. Francis, L. A. Muth, and R. L. Lewis, Proposed uncertainty analysis for RCS measurements, National Institute of Standards and Technology NISTIR 5019, January 1994.

[2] J. R. Taylor, An introduction to error analysis, the study of uncertainties in physical measurements, 2nd Edition, Unversity Science Books, Sausalito, CA, 1982.

[3] G. T. Ruck, D. E. Barrick, W. D. Stuart, and C. K. Krichbaum, Radar Cross Section Handbook. New York: Plenum, 1970.

[4] J. Krauss, Antennas, Second Edition, pp. 587-591, McGraw Hill, New York, 1988.

[5] L. Blake, Radar Range Performance Analysis, pp. 249-253, Artech House, Inc., MA, 1986.

[6] R. L. Lewis, L. A. Muth, and R. C. Wittmann, Polarimetric calibration of reciprocalantenna radars, National Institute of Standards and Technology NISTIR 5033, March 1995.

[7] L. A. Muth, R. L. Lewis, and R. C. Wittmann, Polarimetric calibration of reciprocalantenna radars, AMTA Proceedings, pp. 3-8, Williamsburg, 1995.

[8] L. A. Muth, R. C. Wittmann, and W. Parnell, Polarimetric calibration of nonreciprocal radar systems, AMTA Proceedings, pp. 389-393, Seattle, 1996.

[9] L. A. Muth, Phase dependence in radar cross section measurements, National Institute of Standards and Technology Technical Note 1522, September 2001.

[10] L. A. Muth and T. Conn, Phase-dependent RCS measurements, AMTA Proceedings, pp. 216-221, Cleveland, 2002.

[11] P. T. Boggs and J. E. Rogers, Orthogonal distance regression, Contemporary Mathematics, p. 112, 1990.

[12] P. T. Boggs, R. H. Byrd, J. R. Donaldson, R. B. Schnabel, Algorithm 676 - ODRPACK: Software for weighted orthogonal distance regression, ACM Trans. Math. Software, 15, 1989. 
Appendix A.

INTERPRETATION OF UNCERTAINTY

In the main text we presented a formalism for uncertainty analysis. Here we briefly discuss the motivation for our procedure, especially regarding guidelines presented in a recent NIST publication [2]. For more detail, see [2].

Uncertainties are commonly divided into the categories "random" (type A) and "systematic" (type B). Unfortunately, there seems to be no clear practical distinction between random and systematic, and we have made little attempt to distinguish these types of uncertainties in the main body of this report. We assume that any appropriate actions (such as averaging and background subtraction) already have been taken to mitigate uncertainty. The remaining uncertainties are evaluated as Type B uncertainties.

The treatment of Type B uncertainty in [2] is statistically motivated and is based on a generalized concept of probability [3]. The experimenter assigns a (prior) distribution for each parameter reflecting his or her "personal degree of belief" that the parameter might have some value. We may then apply a probabilistic analysis to estimate combined uncertainty. In this approach, we naturally specify uncertainty in terms of probability, but this probability must be interpreted as a subjective belief and cannot (and must not) be interpreted in the usual random-variable (frequency) sense. To avoid confusion, we have de-emphasized probability, and have simply used the term "reasonable." This practice appropriately begs the question "just what is reasonable, anyway?"

Let us assume that a worst-case uncertainty is equivalent to a 2-sigma uncertainty. [We write out sigma (standard deviation) to distinguish it from $\sigma$ (RCS).] That is, at least for a normal distribution, we are $95 \%$ certain that the error is bounded by our worstcase estimate. Under this hypothesis, the procedure of [2] is essentially identical to ours. However, the NIST document recommends reporting 1-sigma bounds for components of uncertainty and 2-sigma bounds for expanded (overall) uncertainties. In this report we define the worst-case component and overall uncertainties as 2-sigma bounds. 


\section{Appendix B.}

\section{THE EVR RADAR SYSTEM CONFIGURATION, PARAMETERS AND OPERATING CONDITIONS}

The South 40 measurement range (see Figures 1.1 and 1.2) consists of the antenna farm capable of transmitting and receiving all linear polarizations at the frequencies shown in Table B.1. The four environmental Hoffman boxes that house the rf components for the $\mathrm{S}, \mathrm{C}, \mathrm{X}$, and $\mathrm{Ku}$ bands are located close to the antennas in the antenna farm. The rf components in each Hoffman box include a rf converter, transmitters (nominally four per box), and a switch pack. The switch pack contains the HH and VV limiters for the high and low sub-bands, sub-band switching, rf step attenuators, and low noise HH and VV preamplifiers. The receive and transmit components common to these four bands, as well as other radar bands, are located in a nearby temperature-controlled radar room.

The data examined in this report were taken at the South 40 measurement range. The available frequency bands are given in Table B.1. The system configuration and parameters used in the measurements studies in this report are summarized in Tables B.2. The complete set of parameters available for RCS measurements at this range is summarized in Table B.3. A vertical two-way field plot obtained at $11.3 \mathrm{GHz}$ during the cylinder measurements is shown in Figure 4.1.1.

Table B.1. THE EVR Frequency Bands.

\begin{tabular}{ll}
\hline \hline Band & \multicolumn{1}{c}{ Frequency Coverage } \\
HF & $0.03-0.05,0.05-0.1,0.1-0.15$ \\
VHF & $0.150-0.224,0.225-0.299$ \\
UHF & $0.3-0.499,0.5-0.665,0.666-0.999$ \\
& \\
L & $1.0-1.49,1.50-1.99$ \\
S & $2.0-2.99,3.0-3.99$ \\
C & $4.0-5.99,6.0-8.19$ \\
X & $8.2-10.19,10.2-12.39$ \\
Ku & $12.4-14.99,15.0-17.99$ \\
Ka & $34-36$ \\
\hline
\end{tabular}


Table B.2. Typical EVR Outdoor System Configuration for Cylincer Measurements.

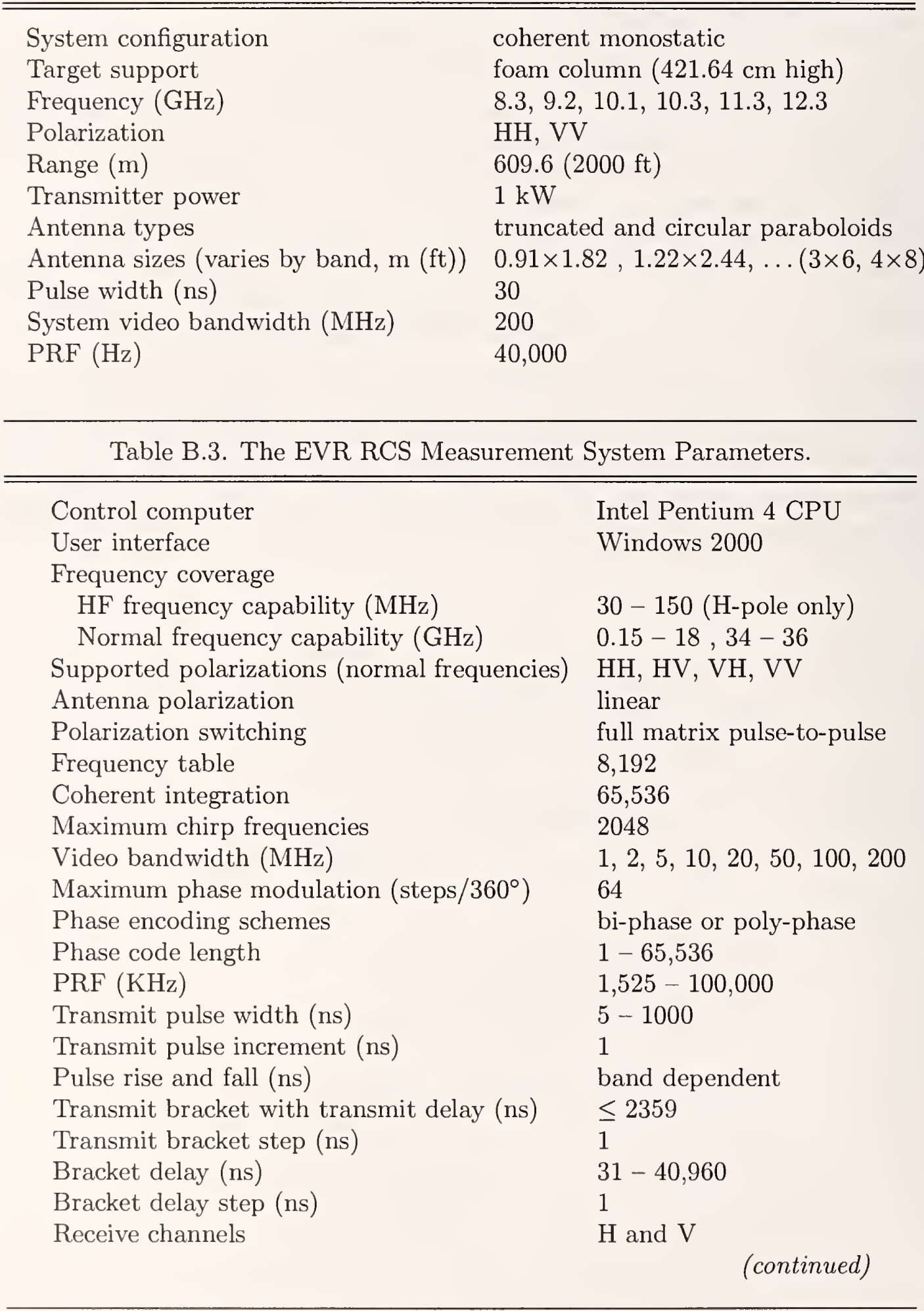


Table B.3. The EVR RCS Measurement System Parameters.

(continued)

Number of range gates

Receive aperture (ps)

Range gate delay (ns)

Monostatic range (m (ft))

Number of frequency bands

IF $(\mathrm{GHz})$

Single pulse dynamic range

I-Q circularity error

System stability $(\mathrm{dB})$

Amplitude linearity

Phase linearity

Phase resolution

Data word length

A/D resolution

$\mathrm{Rx} / \mathrm{Tx}$ attenuation $(\mathrm{dB})$

$\mathrm{Rx} / \mathrm{Tx}$ attenuation step $(\mathrm{dB})$

$\mathrm{RF}$ attenuation $(\mathrm{dB})$

$\mathrm{RF}$ attenuation step (dB)

Pulses in the air

Bias subtraction $(\mathrm{dB})$

Calibration

Data storage interface

Real-time processing

Post-processing

Output media

Simultaneous real time or post processing plots
4 (per receive channel)

100

$31-40,960$

$213,610,1220,1524(700,2000,4000,5000)$

12

2

$>60 \mathrm{~dB}$

$<.2 \mathrm{~dB}$ (real-time corrected)

$+/-0.1$ (after warmup)

$0.2 \mathrm{~dB}$ per $10 \mathrm{~dB}$

$2^{\circ}$ per $10 \mathrm{~dB}$

$0.01^{\circ}$

16 or 32 bit binary

14 bits

$0-63 \mathrm{~dB}$

1

$0-63$

1

single

$\approx-40$

pre- and post-calibration

shared memory

RCS, 1D transforms

global range, RCS, ISAR, doppler, ASCII

medians, statistics, box scores

DVD, CD, hard drive, etc.

8 



\section{NIST Technical Publications}

\section{Periodical}

Journal of Research of the National Institute of Standards and Technology-Reports NIST research and development in metrology and related fields of physical science, engineering, applied mathematics, statistics, biotechnology, and information technology. Papers cover a broad range of subjects, with major emphasis on measurement methodology and the basic technology underlying standardization. Also included from time to time are survey articles on topics closely related to the Institute's technical and scientific programs. Issued six times a year.

\section{Nonperiodicals}

Monographs-Major contributions to the technical literature on various subjects related to the Institute's scientific and technical activities.

Handbooks-Recommended codes of engineering and industrial practice (including safety codes) devel- oped in cooperation with interested industries, professional organizations, and regulatory bodies.

Special Publications-Include proceedings of conferences sponsored by NIST, NIST annual reports, and other special publications appropriate to this grouping such as wall charts, pocket cards, and bibliographies.

National Standard Reference Data Series-Provides quantitative data on the physical and chemical properties of materials, compiled from the world's literature and critically evaluated. Developed under a worldwide program coordinated by NIST under the authority of the National Standard Data Act (Public Law 90-396). NOTE: The Journal of Physical and Chemical Reference Data (JPCRD) is published bimonthly for NIST by the American Institute of Physics (AIP). Subscription orders and renewals are available from AIP, P.O. Box 503284, St. Louis, MO 63150-3284.

Building Science Series-Disseminates technical information developed at the Institute on building materials, components, systems, and whole structures. The series presents research results, test methods, and performance criteria related to the structural and environmental functions and the durability and safety characteristics of building elements and systems.

Technical Notes-Studies or reports which are complete in themselves but restrictive in their treatment of a subject. Analogous to monographs but not so comprehensive in scope or definitive in treatment of the subject area. Often serve as a vehicle for final reports of work performed at NIST under the sponsorship of other government agencies. Voluntary Product Standards-Developed under procedures published by the Department of Commerce in Part 10, Title 15, of the Code of Federal Regulations. The standards establish nationally recognized requirements for products, and provide all concerned interests with a basis for common understanding of the characteristics of the products. NIST administers this program in support of the efforts of private-sector standardizing organizations.

Order the following NIST publications-FIPS and NISTIRs-from the National Technical Information Service, Springfield, VA 22161.

Federal Information Processing Standards Publications (FIPS PUB)-Publications in this series collectively constitute the Federal Information Processing Standards Register. The Register serves as the official source of information in the Federal Government regarding standards issued by NIST pursuant to the Federal Property and Administrative Services Act of 1949 as amended, Public Law 89-306 (79 Stat. 1127), and as implemented by Executive Order 11717 (38 FR 12315, dated May 11,1973) and Part 6 of Title 15 CFR (Code of Federal Regulations).

NIST Interagency or Internal Reports (NISTIR)-The series includes interim or final reports on work performed by NIST for outside sponsors (both government and nongovernment). In general, initial distribution is handled by the sponsor; public distribution is handled by sales through the National Technical Information Service, Springfield, VA 22161, in hard copy, electronic media, or microfiche form. NISTIR's may also report results of NIST projects of transitory or limited interest, including those that will be published subsequently in more comprehensive form. 
U.S. Department of Commerce

National Bureau of Standards and Technology

325 Broadway

Boulder, CO 80305-3328

Official Business

Penalty for Private Use $\$ 300$ 